\title{
FROM RESOLVENT ESTIMATES TO UNIQUE CONTINUATION FOR THE SCHRÖDINGER EQUATION
}

\author{
IHYEOK SEO
}

\begin{abstract}
In this paper we develop an abstract method to handle the problem of unique continuation for the Schrödinger equation $\left(i \partial_{t}+\Delta\right) u=V(x) u$. In general the problem is to find a class of potentials $V$ which allows the unique continuation. The key point of our work is to make a direct link between the problem and the weighted $L^{2}$ resolvent estimates $\left\|(-\Delta-z)^{-1} f\right\|_{L^{2}(|V|)} \leq$ $C\|f\|_{L^{2}\left(|V|^{-1}\right)}$. We carry it out in an abstract way, and thereby we do not need to deal with each of the potential classes. To do so, we will make use of the limiting absorption principle and Kato $H$-smoothing theorem in spectral theory, and employ some tools from harmonic analysis. Once the resolvent estimate is set up for a potential class, from our abstract theory the unique continuation would follow from the same potential class. Also, it turns out that there can be no dented surface on the boundary of the maximal open zero set of the solution $u$. In this regard, another main issue for us is to know which class of potentials allows the resolvent estimate. We establish such a new class which contains previously known ones, and we will also apply it to the problem of well-posedness for the equation.
\end{abstract}

\section{INTRODUCTION AND MAIN RESULTS}

It is well known that an analytic function has a property of unique continuation which says that it cannot vanish in any non-empty open subset of its domain of definition without being identically zero. The property results from expanding the function in power series because it would vanish with all its derivatives at some point in the subset. At this point, we can relate the property to a solution of the Cauchy-Riemann equation $\bar{\partial} u=0$ in $\mathbb{R}^{2}$, since it should be complex analytic. Similarly, the same property holds for solutions to the Laplace equation $\Delta u=0$ in $\mathbb{R}^{n}, n \geq 2$, since they are harmonic functions that are still real analytic. Now it can be asked whether the property is shared by other partial differential equations whose solutions are not necessarily analytic, or even smooth. It would be an interesting problem to prove the property for such an equation.

This paper is mainly concerned with the problem for solutions of the Schrödinger equation

$$
i \partial_{t} \Psi(x, t)=(-\Delta+V(x)) \Psi(x, t)
$$

which describes how the wave function $\Psi$ of a non-relativistic quantum mechanical system with a potential $V$ evolves over time. In principle, the unique continuation

Received by the editors September 3, 2014 and, in revised form, November 2, 2015 and November 15, 2014.

2010 Mathematics Subject Classification. Primary 47A10, 35B60; Secondary 35Q40.

Key words and phrases. Resolvent estimates, unique continuation, Schrödinger equations. 
can be viewed as one of the non-localization properties of the wave function which are a fruitful issue in certain interpretations of quantum mechanics.

Let us first put (1.1) in a reasonably general setting which is a differential inequality of the form

$$
\left|\left(i \partial_{t}+\Delta\right) u(x, t)\right| \leq|V(x) u(x, t)|,
$$

where $u: \mathbb{R}^{n+1} \rightarrow \mathbb{C}$ is a solution that is a function satisfying (1.2) and $V: \mathbb{R}^{n} \rightarrow \mathbb{C}$ is a potential. From now on, we call (1.2) a Schrödinger inequality for convenience. Let us also make precise what we will mean by unique continuation property:

Definition 1.1. Given a partial differential equation or an inequality in $\mathbb{R}^{n}$, we say that it has the unique continuation property from a non-empty open subset $\Omega \subset \mathbb{R}^{n}$ if its solution cannot vanish in $\Omega$ without being identically zero.

The general question to ask is for which class of potentials does the unique continuation hold. It has been studied for decades with a half-space $\Omega$ in $\mathbb{R}^{n+1}$. Apart from the case (1.2), the case of time-dependent potentials $V(x, t)$ was mainly studied in [22, 27, 35, 36]. The key ingredients in these works are the so-called Carleman estimates 1 for the operator $i \partial_{t}+\Delta$. The first one, due to Kenig and Sogge [22], is the estimate

$$
\left\|e^{\beta\langle(x, t), \nu\rangle} u\right\|_{L_{t, x}^{\frac{2(n+2)}{n}}\left(\mathbb{R}^{n+1}\right)} \leq C\left\|e^{\beta\langle(x, t), \nu\rangle}\left(i \partial_{t}+\Delta\right) u\right\|_{L_{t, x}^{\frac{2(n+2)}{n+4}}}{ }_{\left(\mathbb{R}^{n+1}\right)},
$$

where $\langle$,$\rangle denotes the usual inner product on \mathbb{R}^{n+1}$ and the constant $C$ is independent of $\beta \in \mathbb{R}$ and $\nu \in \mathbb{R}^{n+1}$. Note that the special case where $\beta=0$ becomes equivalent to an estimate of Strichartz [41] for the inhomogeneous Schrödinger equation. In this regard, later developments [17, 27, 36] have been made to extend (1.3) to mixed norms $L_{t}^{q} L_{x}^{r}\left(L_{t}^{q}\left(\mathbb{R} ; L_{x}^{r}\left(\mathbb{R}^{n}\right)\right)\right.$ for which the inhomogeneous Strichartz estimate is known to hold (as in [14, 20, 44]). This made it possible to obtain the unique continuation for potentials $V \in L_{t}^{p} L_{x}^{s}$ where $(p, s)$ lies in the scaling-critical range $2 / p+n / s=2$ (see [27,36]). In a similar manner, these works were further extended in 35 to Wiener amalgam norms which may be of interest since they are not allowed to possess a scaling invariance ([10]).

Unfortunately the above-mentioned Carleman estimates are no longer available in the time-independent case (1.2). The aim of this paper is to develop an abstract method to handle the unique continuation problem for (1.2) and to exhibit a few useful applications of this method. One of our main contributions is to convert the problem to that of obtaining the following resolvent estimate on weighted $L^{2}$ spaces:

$$
\left\|(-\Delta-z)^{-1} f\right\|_{L^{2}(|V|)} \leq C(V)\|f\|_{L^{2}\left(|V|^{-1}\right)},
$$

where $z \in \mathbb{C} \backslash \mathbb{R}$ and $C(V)$ is a suitable constant depending on the potential $V(x)$ but should also be independent of $z$. At this point, it should be noted that our method focuses more on the goal of obtaining unique continuation directly from resolvent estimates rather than Carleman estimates.

We shall carry out the project in a unified manner, and thereby we do not need to deal with each of the potentials which allow (1.4). Let us first make the following

\footnotetext{
${ }^{1}$ The Carleman method, which derives unique continuation from the Carleman estimate, originated from the pioneering work of Carleman [6] for elliptic equations.
} 
definition in which we denote $A \sim B$ to mean $C A \leq B \leq C A$ with unspecified constants $C>0$.

Definition 1.2. We say that a potential class $\mathcal{R}$ is of resolvent type if there exists a suitable function $[\cdot]_{\mathcal{R}}$ depending only on $\mathcal{R}$ itself such that the resolvent estimate (1.4) holds with bound $[V]_{\mathcal{R}}$.

Remark 1.3. Note that if $\left|V_{1}\right| \leq\left|V_{2}\right|$ and (1.4) holds for $V_{2}$, then it also holds for $V_{1}$ with $C\left(V_{1}\right)=C\left(V_{2}\right)$. In this case, we take $\left[V_{1}\right]_{\mathcal{R}}$ to be less than or equal to $\left[V_{2}\right]_{\mathcal{R}}$.

Here and thereafter, we always use the letter $\mathcal{R}$ to mean a potential class of resolvent type. Once a suitable class $\mathcal{R}$ is set up, from our abstract theory the unique continuation for the Schrödinger inequality would follow from $V \in \mathcal{R}$. At this point, the main issue for us is to know which class of potentials can be of resolvent type. As we will see later, the scaling-critical Lebesgue class $L^{n / 2}$, with $[\cdot]_{L^{n / 2}}=\|\cdot\|_{L^{n / 2}}$, is one of such classes. In fact we establish a new and wider class which contains $L^{n / 2, \infty}$ and even the Fefferman-Phong class $\mathcal{L}^{2, p}$ (see (2.1)) for $p>(n-1) / 2, n \geq 3$. In particular, when $n=3$ it also contains the global Kato and Rollnik classes (see (2.5), (2.6) ). These will be discussed in detail in the next section as one of the cores of our work.

Before stating our results on unique continuation, we need to set up more notation in order to be precise. A weight 2$]: \mathbb{R}^{n} \rightarrow[0, \infty]$ is said to be of Muckenhoupt $A_{p}\left(\mathbb{R}^{n}\right)$ class, $1<p<\infty$, if there is a constant $C_{A_{p}}$ such that

$$
\sup _{Q \text { cubes in } \mathbb{R}^{n}}\left(\frac{1}{|Q|} \int_{Q} w(x) d x\right)\left(\frac{1}{|Q|} \int_{Q} w(x)^{-\frac{1}{p-1}} d x\right)^{p-1}<C_{A_{p}} .
$$

Note that $w \in A_{2} \Leftrightarrow w^{-1} \in A_{2}$. Given $v \in \mathbb{R}^{n}$, one may write $x=s v+\widetilde{x}$ for $x \in \mathbb{R}^{n}$, where $s \in \mathbb{R}$ and $\widetilde{x}$ is in some hyperplane $\mathcal{P}$ whose normal vector is $v$. We shall denote $w \in A_{p}(v)$ to mean that $w$ is in the $A_{p}$ class in the onedimensional direction of the vector $v$ if the function $w_{\widetilde{x}}(s):=w(x)$ is in $A_{p}(\mathbb{R})$ with $C_{A_{p}}$ uniformly in almost every $\widetilde{x} \in \mathcal{P}$. At first glance this notion could be more or less complicated. But, by translation and rotation it can be reduced to the case where $v=(0, \ldots, 0,1) \in \mathbb{R}^{n}$ and $\mathcal{P}=\mathbb{R}^{n-1}$. In this case $w \in A_{p}(v)$ means that $w\left(x_{1}, \ldots, x_{n-1}, \cdot\right) \in A_{p}(\mathbb{R})$ in the $x_{n}$ variable uniformly in almost every $\widetilde{x}=\left(x_{1}, \ldots, x_{n-1}\right) \in \mathbb{R}^{n-1}$. We point out that this one-dimensional $A_{p}$ condition is trivially satisfied if $w$ is in a more restrictive $A_{p}\left(\mathbb{R}^{n}\right)$ class defined over arbitrary rectangles instead of cubes (see Lemma 2.2 in [25]). For $\nu=\left(\nu_{1}, \ldots, \nu_{n}, \nu_{n+1}\right) \in$ $\mathbb{R}^{n+1}$, we denote by $\nu^{\prime}$ the vector $\left(\nu_{1}, \ldots, \nu_{n}\right)$ in $\mathbb{R}^{n}$.

Let us now state our unique continuation theorems for the Schrödinger inequality

$$
\left|\left(i \partial_{t}+\Delta\right) u(x, t)\right| \leq|V(x) u(x, t)|,
$$

where $V \in \mathcal{R}$. There are two types of these theorems, global and local. First, the following global theorem says that if the solution of (1.6) is supported on one side of a hyperplane in $\mathbb{R}^{n+1}$, then it must vanish on all of $\mathbb{R}^{n+1}$. In other words, the unique continuation arises globally from a half-space in $\mathbb{R}^{n+1}$.

Theorem 1.4. Let $n \geq 2$. Assume that $u \in \mathcal{H}_{t}^{1}\left(\mathcal{L}_{2}\right) \cap \mathcal{H}_{x}^{2}\left(\mathcal{L}_{2}\right)$ is a solution of (1.6) with $V \in \mathcal{R}$ and vanishes in a half-space with a unit normal vector $\nu \in \mathbb{R}^{n+1}$. Then

\footnotetext{
${ }^{2}$ It is a locally integrable function which is allowed to be zero or infinite only on a set of Lebesgue measure zero, so $w^{-1}$ is also a weight if it is locally integrable.
} 
it must be identically zero if $|V| \in A_{2}\left(\nu^{\prime}\right)$ and $[V]_{\mathcal{R}}<\varepsilon$ for a sufficiently small $\varepsilon>0$.

Let us give more details about the assumptions in the theorem. First, $\mathcal{L}_{2}=$ $L^{2} \cap L^{2}\left(|V|^{-1}\right)$ is the solution space for which we have unique continuation, and $\mathcal{H}_{t}^{1}\left(\mathcal{L}_{2}\right)$ denotes the space of functions whose derivatives up to order 1 , with respect to the time variable $t$, belong to $\mathcal{L}_{2}$. Similarly for $\mathcal{H}_{x}^{2}\left(\mathcal{L}_{2}\right)$. It should be noted that the solution space is dense in $L^{2}$. In fact, consider $D_{n}=\left\{x \in \mathbb{R}^{n}:|V|^{-1 / 2} \leq n\right\}$. Then, for $f \in L^{2}$ the function $\chi_{D_{n}} f$ is contained in $\mathcal{L}_{2}$, and $\chi_{D_{n}} f \rightarrow f$ as $n \rightarrow \infty$. By the Lebesgue dominated convergence theorem, it now follows that $\chi_{D_{n}} f \rightarrow f$ in $L^{2}$. So, $\mathcal{L}_{2}$ is dense in $L^{2}$.

Next, the smallness assumption such as $[V]_{\mathcal{R}}<\varepsilon$ is quite standard in the study of unique continuation. In some cases of $\mathcal{R}$ where the assumption $|V| \in A_{2}\left(\nu^{\prime}\right)$ is superfluous, it can be replaced by a more local one,

$$
\sup _{a \in \mathbb{R}} \lim _{\delta \rightarrow 0}\left[\chi_{S_{a, a+\delta}\left(\nu^{\prime}\right)} V\right]_{\mathcal{R}}<\varepsilon \quad\left(\text { if } \nu=\left(\nu^{\prime}, 0\right)\right),
$$

which is trivially satisfied for the case $\mathcal{R}=L^{n / 2}$. Here $\chi_{E}$ denotes the characteristic function of a set $E$ in $\mathbb{R}^{n}$, and for $a \in \mathbb{R}$ and a unit vector $v \in \mathbb{R}^{n}, S_{a, a+\delta}(v)$ denotes a "strip" in $\mathbb{R}^{n}$ with width $\delta>0$ given by

$$
S_{a, a+\delta}(v):=\left\{x \in \mathbb{R}^{n}: a<\langle x, v\rangle \leq a+\delta\right\} .
$$

Also, the solution space can be extended to the whole space $L^{2}$ in such cases. See Section 6 for details.

Now we turn to another type of unique continuation which is more local in nature. First we point out that there exists a smooth potential $V$ such that $\left(i \partial_{t}+\Delta\right) u=$ $V(x, t) u, 0 \in \operatorname{supp} u$, and $u=0$ on $\left\{(x, t) \in \mathbb{R}^{n+1}: x_{1}<0\right\}$ in a neighborhood of the origin. This result is from a particular case of Théorème 1.6 in [26] due to Lascar and Zuily. (See also 45, p. 127, Theorem 2.10.) In this case the solution $u$ cannot vanish near the origin across the hyperplane $\left\{(x, t) \in \mathbb{R}^{n+1}: x_{1}=0\right\}$ because $0 \in \operatorname{supp} u$. This shows that the Schrödinger equation does not have, as a rule, a property of unique continuation locally across a hyperplane in $\mathbb{R}^{n+1}$. But, our result below says that the unique continuation can arise locally across a hypersurface on a sphere in $\mathbb{R}^{n+1}$ into an interior region of the sphere.

Theorem 1.5. Let $n \geq 2$ and let $S_{r}^{n}$ be a sphere in $\mathbb{R}^{n+1}$ with radius $r$. Assume that $u \in \mathcal{H}_{t}^{1}\left(\mathcal{L}_{2}\right) \cap \mathcal{H}_{x}^{2}\left(\mathcal{L}_{2}\right)$ is a solution of (1.6) with $V \in \mathcal{R}$ and vanishes on an exterior neighborhood of $S_{r}^{n}$ in a neighborhood of a point $p \in S_{r}^{n}$. Let $\nu$ be the unit outward normal vector of $S_{r}^{n}$ at $p$. Then it follows that $u \equiv 0$ in a neighborhood of $p$, if $|V| \in A_{2}\left(\nu^{\prime}\right)$ and $[V]_{\mathcal{R}}<\varepsilon$ for a sufficiently small $\varepsilon>0$.

In the same cases as above, the solution space $\mathcal{L}_{2}$ can be extended to the whole space $L^{2}$, and the smallness assumption can be replaced by a more local one,

$$
\lim _{\delta \rightarrow 0}\left[\chi_{B_{\delta}\left(p^{\prime}\right)} V\right]_{\mathcal{R}}<\varepsilon
$$

where $B_{\delta}(p)$ denotes a ball centered at $p$ with radius $\delta$.

More interestingly, the above local theorem gives us a possibility of seeing the geometric shape of the zero set of the solutions. Roughly speaking, there can be no dented surface on the boundary of the maximal open zero set. Let us first introduce the following definition. 
Definition 1.6. Let $n \geq 2$. Then we say that a non-empty open set $\Omega \varsubsetneqq \mathbb{R}^{n}$ has a dent at a point $p$ in the boundary $\partial \Omega$ if there is a sphere $S_{r}^{n-1}$ in $\mathbb{R}^{n}$ such that $p \in S_{r}^{n-1}$ and an exterior neighborhood of $S_{r}^{n-1}$ in a neighborhood of $p$ is contained in $\Omega$.

The following corollary is now deduced from the above local unique continuation.

Corollary 1.7. Let $\mathcal{M}$ be the maximal open set in $\mathbb{R}^{n+1}$ on which the solution $u$ of (1.6) with $V \in \mathcal{R}$ vanishes. Then the boundary $\partial \mathcal{M}$ cannot have a dent if the same assumptions as in Theorem 1.5 hold.

Proof. Indeed, if there is a dent at $p \in \partial \mathcal{M}$, then it is clear that $u$ vanishes on an exterior neighborhood of a sphere $S_{r}^{n}$ in a neighborhood of $p$. From the above local unique continuation, $u$ must vanish in a neighborhood of $p$, and so $p \in \mathcal{M}$. But this contradicts the maximality of $M$.

Finally, let us sketch the organization of the paper. In Section 2 we establish a new function class of $V$ which allows the resolvent estimate (1.4). Then, new results on the unique continuation are immediately obtained from the abstract global and local theorems that are proved in Sections 6 and 7 respectively. The key ingredient in the proof is the abstract Carleman estimate

$$
\left\|e^{\beta\langle(x, t), \nu\rangle} u\right\|_{L_{t, x}^{2}(|V|)} \leq C[V]\left\|e^{\beta\langle(x, t), \nu\rangle}\left(i \partial_{t}+\Delta\right) u\right\|_{L_{t, x}^{2}\left(|V|^{-1}\right)},
$$

where $C$ is a constant independent of $\beta \in \mathbb{R}$ and $\nu \in \mathbb{R}^{n+1}$, and $[V]$ denotes the least constant $C(V)$ for which the resolvent estimate (1.4) holds. In Section 5 we will show (1.7), which means that a potential for the resolvent estimate can be made to work for the Carleman estimate. In fact our basic strategy is to derive (1.7) only from (1.4) in an abstract way. This is done in several steps and based on the weighted $L^{2}$ estimate

$$
\left\|\int_{-\infty}^{\infty} e^{i(t-s) \Delta} F(\cdot, s) d s\right\|_{L_{t, x}^{2}(|V|)} \leq C C(V)\|F\|_{L_{t, x}^{2}\left(|V|^{-1}\right)},
$$

where $e^{i t \Delta}$ is the free Schrödinger propagator (see (4.1)) and $C(V)$ is the constant in the resolvent estimate (1.4). In Section 4 we derive (1.8) only from (1.4) in two ways. The first is a concrete one using a Fourier restriction estimate which results from a limiting absorption principle obtained in Section 3. On the other hand, the second is a more direct one appealing to Kato $H$-smoothing theory. Our new resolvent estimates may have further applications for other related problems. In fact we will apply them in Section 8 to the problem of well-posedness for the Schrödinger equation. The method in this paper can also be applied to the case of time-dependent potentials $V(x, t)$ such that

$$
\sup _{t \in \mathbb{R}}|V(x, t)| \leq W(x) \in \mathcal{R} .
$$

This takes up the final section, Section 9 .

Throughout this paper, the letter $C$ stands for positive constants possibly different at each occurrence. We also denote by $\widehat{f}$ and $\mathcal{F}^{-1}(f)$ the Fourier and the inverse Fourier transforms of $f$, respectively. 


\section{Resolvent estimates}

The aim of this section is twofold: firstly to look at which class of potentials can be of resolvent type, and secondly to establish such a new class, extending and generalizing those in [8] and [3, respectively.

Let us consider the resolvent $(-\Delta-z)^{-1}$ for $z \in \mathbb{C} \backslash \mathbb{R}$. We shall use the standard notation $R_{0}(z)=(-\Delta-z)^{-1}$ for convenience. (See the next section for details.)

We start with the scaling-critical Lebesgue class $L^{n / 2}$. Indeed, by the scaling $(x, t) \rightarrow\left(\lambda x, \lambda^{2} t\right), u_{\lambda}(x, t)=u\left(\lambda x, \lambda^{2} t\right)$ takes the equation $i \partial_{t} u+\Delta u=V(x) u$ into $i \partial_{t} u_{\lambda}+\Delta u_{\lambda}=V_{\lambda}(x) u_{\lambda}$, where $V_{\lambda}(x)=\lambda^{2} V(\lambda x)$. Thus, $\left\|V_{\lambda}\right\|_{L^{p}}=\lambda^{2-n / p}\|V\|_{L^{p}}$ and the $L^{p}$ norm of $V_{\lambda}$ is independent of $\lambda$ precisely when $p=n / 2$. In what follows, it will be convenient to keep in mind that a potential class is said to be scaling invariant if it is invariant under the scaling $V_{\lambda}(x)=\lambda^{2} V(\lambda x)$ forced by the Schrödinger equation onto the potential $V$ as above. As we will see below, the class $L^{n / 2}$ is of resolvent type with $[\cdot]_{L^{n / 2}}=\|\cdot\|_{L^{n / 2}}$, but it is too small to contain the inverse square potential $V(x)=a /|x|^{2}(a>0)$ which allows the resolvent estimate,

$$
\left\|R_{0}(z) f\right\|_{L^{2}\left(a /|x|^{2}\right)} \leq C a\|f\|_{L^{2}\left(|x|^{2} / a\right)},
$$

due to Kato and Yajima 19. This potential has attracted considerable interest from mathematical physics. This is because the Schrödinger operator $-\Delta+a /|x|^{2}$ is physically related to the Hamiltonian of a spin-zero quantum particle in a Coulomb field ([7]) and behaves very differently depending on the value of the constant $a$ ( $c f$. 30, 31]).

Now we consider a wider class of resolvent type where we can consider singularities of the type $a /|x|^{2}$. Let $\mathcal{L}^{\alpha, p}$ denote the Morrey-Campatano class which is defined for $\alpha>0$ and $1 \leq p \leq n / \alpha$ by

$$
V \in \mathcal{L}^{\alpha, p} \Leftrightarrow\|V\|_{\mathcal{L}^{\alpha, p}}:=\sup _{Q \text { cubes in } \mathbb{R}^{n}}|Q|^{\alpha / n}\left(\frac{1}{|Q|} \int_{Q}|V(y)|^{p} d y\right)^{1 / p}<\infty .
$$

Then the case $\alpha=2$ is of special interest for us, since $\mathcal{L}^{2, n / 2}=L^{n / 2}$ and $a /|x|^{2} \in$ $L^{n / 2, \infty} \subset \mathcal{L}^{2, p}$ if $p<n / 2$. Also, $\mathcal{L}^{2, p}$ is the only possible scaling-invariant MorreyCampatano class because $\|V(\lambda x)\|_{\mathcal{L}^{\alpha, p}}=\lambda^{-\alpha}\|V\|_{\mathcal{L}^{\alpha, p}}$. This special class is sometimes called the Fefferman-Phong class since it was introduced by C. Fefferman and D. H. Phong regarding spectral properties of the Schrödinger operator $-\Delta+V(x)$. Thanks to a result of Chanillo and Sawyer [8], the Fefferman-Phong class is of resolvent type with $[\cdot]_{\mathcal{L}^{2, p}}=\|\cdot\|_{\mathcal{L}^{2, p}}$ in some range of $p$. Precisely, for $p>(n-1) / 2$, $n \geq 3$,

$$
\left\|R_{0}(z) f\right\|_{L^{2}(|V|)} \leq C\|V\|_{\mathcal{L}^{2, p}}\|f\|_{L^{2}\left(|V|^{-1}\right)}
$$

The aim here is to extend (2.2) to a new and wider class of potentials. First we need to introduce some notation. We say that $V$ is in the Kerman-Sawyer class $\mathcal{K S}_{\alpha}$ for $0<\alpha<n$ if

$$
\|V\|_{\mathcal{K} \mathcal{S}_{\alpha}}:=\sup _{Q}\left(\int_{Q}|V(x)| d x\right)^{-1} \int_{Q} \int_{Q} \frac{|V(x) V(y)|}{|x-y|^{n-\alpha}} d x d y<\infty .
$$


Here the sup is taken over all dyadic cubes $Q$ in $\mathbb{R}^{n}$. Our initial motivation for (2.3) stemmed from finding all the possible potentials $V(x)$ which allow the socalled Fefferman-Phong inequality

$$
\int_{\mathbb{R}^{n}}|u|^{2}|V| d x \leq C_{V} \int_{\mathbb{R}^{n}}|\nabla u|^{2} d x
$$

where $C_{V}$ is a suitable constant depending on $V$. As is well known from [13, (2.4) holds for $V \in \mathcal{L}^{2, p}, 1<p \leq n / 2$, with $C_{V} \sim\|V\|_{\mathcal{L}^{2, p}}$, but it is not valid for $p=1$ as remarked in [12. As a result of Kerman and Sawyer [24] (see (2.12) below), the least constant $C_{V}$ for which (2.4) holds may be taken to be a constant multiple of the norm $\|V\|_{\mathcal{K S}}$, and so $\mathcal{L}^{2, p} \subset \mathcal{K} \mathcal{S}_{2}$ if $p \neq 1$. In general, $\mathcal{L}^{\alpha, p} \subset \mathcal{K} \mathcal{S}_{\alpha}$ if $p \neq 1$ (see [3], Subsection 2.2). Now we define a new function class which contains $\mathcal{L}^{2, p}$ for all $p>(n-1) / 2, n \geq 3$.

Definition 2.1. Let $n \geq 3$. We say that $V$ is in the class $\mathcal{S}_{n}$ if $V^{\frac{n-1}{2}} \in \mathcal{K} \mathcal{S}_{n-1}$, and define a quantity $[\cdot]_{\mathcal{S}_{n}}$ on $\mathcal{S}_{n}$ by

$$
[V]_{\mathcal{S}_{n}}:=\left\|V^{\frac{n-1}{2}}\right\|_{\mathcal{K S}_{n-1}^{n-1}}^{\frac{2}{n-1}}
$$

First, note that $\mathcal{S}_{n}$ is just the same as $\mathcal{K S}_{2}$ when $n=3$. In this case $\mathcal{S}_{n}$ is also closely related to the global Kato and Rollnik classes which are defined by

$$
V \in \mathcal{K} \quad \Leftrightarrow \quad\|V\|_{\mathcal{K}}:=\sup _{x \in \mathbb{R}^{3}} \int_{\mathbb{R}^{3}} \frac{|V(y)|}{|x-y|} d y<\infty
$$

and

$$
V \in \mathcal{R} \quad \Leftrightarrow \quad\|V\|_{\mathcal{R}}:=\int_{\mathbb{R}^{3}} \int_{\mathbb{R}^{3}} \frac{|V(x) V(y)|}{|x-y|^{2}} d x d y<\infty,
$$

respectively. These are fundamental ones in spectral and scattering theory ( $c f$. [18, 38]), and their usefulness for dispersive properties of the Schrödinger equation was revealed in the recent work 32 of Rodnianski and Schlag. It is an elementary matter to check that $\mathcal{K} \subset \mathcal{S}_{3}$ and $\mathcal{R} \subset \mathcal{S}_{3}$. Also it is easy to see that $\left\|V^{m}\right\|_{\mathcal{L}^{\alpha, p}}=$ $\|V\|_{\mathcal{L}^{\alpha / m, m p}}^{m}$ for $m>0$. If this still holds for $\mathcal{K} \mathcal{S}_{\alpha}$, then $\mathcal{S}_{n}$ would become equivalent to $\mathcal{K S}_{2}$ for all dimensions. But such property does not carry over to $\mathcal{K} \mathcal{S}_{\alpha}$ even though $\mathcal{L}^{\alpha, p} \subset \mathcal{K} \mathcal{S}_{\alpha}$. Next, we point out that the class $\mathcal{S}_{n}$ is wider than $\mathcal{L}^{2, p}$ for all $p>(n-1) / 2$. Indeed, if $V \in \mathcal{L}^{2, p}$, then $V^{\frac{n-1}{2}} \in \mathcal{L}^{n-1,2 p /(n-1)} \subset \mathcal{K} \mathcal{S}_{n-1}$ when $2 p /(n-1)>1$, and

$$
[V]_{\mathcal{S}_{n}}=\left\|V^{\frac{n-1}{2}}\right\|_{\mathcal{K S}_{n-1}^{n-1}}^{\frac{2}{n-1}} \leq\left\|V^{\frac{n-1}{2}}\right\|_{\mathcal{L}^{n-1,2 p /(n-1)}}^{\frac{2}{n-1}}=\|V\|_{\mathcal{L}^{2, p}}
$$

Finally, it should be noted that $\mathcal{S}_{n}$ is scaling invariant with respect to $[\cdot]_{\mathcal{S}_{n}}$ :

$$
\left[\lambda^{2} V(\lambda x)\right]_{\mathcal{S}_{n}}=\lambda^{2}\left\|V(\lambda x)^{\frac{n-1}{2}}\right\|_{\mathcal{K S}_{n-1}}^{\frac{2}{n-1}}=\lambda^{2}\left(\lambda^{-(n-1)}\left\|V^{\frac{n-1}{2}}\right\|_{\mathcal{K S} \mathcal{S}_{n-1}}\right)^{\frac{2}{n-1}}=[V]_{\mathcal{S}_{n}} .
$$

Now we are ready to state the following result extending (2.2) to the class $\mathcal{S}_{n}$.

Theorem 2.2. Let $n \geq 3$. Then the class $\mathcal{S}_{n}$ is of resolvent type. Namely, for $V \in \mathcal{S}_{n}$

$$
\left\|R_{0}(z) f\right\|_{L^{2}(|V|)} \leq C[V]_{\mathcal{S}_{n}}\|f\|_{L^{2}\left(|V|^{-1}\right)}
$$

with a constant $C$ independent of $z \in \mathbb{C} \backslash \mathbb{R}$. 
Remark 2.3. It is worth comparing with Theorem 2.2 in [3, which proves (2.7) only for $n=3$ with a different approach that does not work for higher dimensions $n \geq 4$.

Proof of Theorem 2.2. By scaling we may first assume that $|z|=1$. Indeed, note that

$$
R_{0}(z) f(x)=(-\Delta-z)^{-1} f(x)=|z|^{-1}(-\Delta-z /|z|)^{-1}\left[f\left(|z|^{-1 / 2} \cdot\right)\right]\left(|z|^{1 / 2} x\right) .
$$

So, if (2.7) holds for $|z|=1$, then for $z \in \mathbb{C} \backslash \mathbb{R}$,

$$
\begin{aligned}
\left\|R_{0}(z) f\right\|_{L^{2}(|V|)}^{2} & =|z|^{-2}|z|^{-n / 2}\left\|R_{0}(z /|z|)\left[f\left(|z|^{-1 / 2} \cdot\right)\right]\right\|_{L^{2}\left(\left|V\left(|z|^{-1 / 2} \cdot\right)\right|\right)}^{2} \\
& \leq C|z|^{-2}|z|^{-n / 2}\left[V\left(|z|^{-1 / 2} \cdot\right)\right]_{\mathcal{S}_{n}}^{2}\left\|f\left(|z|^{-1 / 2} \cdot\right)\right\|_{L^{2}\left(\left|V\left(|z|^{-1 / 2} \cdot\right)\right|^{-1}\right)}^{2} \\
& \leq C[V]_{\mathcal{S}_{n}}^{2}\|f\|_{L^{2}\left(|V|^{-1}\right)}^{2} .
\end{aligned}
$$

Now we rewrite (2.7) in the equivalent form,

$$
\left\||V|^{1 / 2} R_{0}(z)\left(|V|^{1 / 2} f\right)\right\|_{L^{2}} \leq C[V]_{\mathcal{S}_{n}}\|f\|_{L^{2}},
$$

and will show this using Stein's complex interpolation ( $c f$. 40]), as in 8, 21, on an analytic family of operators $T_{\lambda}$ defined for $\lambda \in \mathbb{C}$ by

$$
T_{\lambda}=|V|^{\lambda / 2}(-\Delta-z)^{-\lambda}|V|^{\lambda / 2}
$$

with the principal branch. First, from Plancherel's theorem we have the trivial estimate for $\operatorname{Re}(\lambda)=0$ :

$$
\left\|T_{\lambda} f\right\|_{L^{2}} \leq e^{\pi|\operatorname{Im}(\lambda)|}\|f\|_{L^{2}} .
$$

In fact, since $|V|^{\lambda / 2}=1$ for $\operatorname{Re}(\lambda)=0$, from Plancherel's theorem we see that

$$
\begin{aligned}
\left\|T_{\lambda} f\right\|_{L^{2}} & =\left\|\frac{1}{\left(|\xi|^{2}-z\right)^{\lambda}} \widehat{f}(\xi)\right\|_{L^{2}} \\
& \leq \sup _{\xi \in \mathbb{R}^{n}}\left|\frac{1}{\left(|\xi|^{2}-z\right)^{\lambda}}\right|\|f\|_{L^{2}} \\
& \leq \sup _{\xi \in \mathbb{R}^{n}} e^{\operatorname{Im}(\lambda) \arg \left(|\xi|^{2}-z\right)}\|f\|_{L^{2}} \\
& \leq e^{\pi|\operatorname{Im}(\lambda)|}\|f\|_{L^{2}} .
\end{aligned}
$$

On the other hand, we will get for $\operatorname{Re}(\lambda)=(n-1) / 2$,

$$
\left\|T_{\lambda} f\right\|_{L^{2}} \leq C e^{\frac{\pi}{2}|\operatorname{Im}(\lambda)|}\left\|V^{\frac{n-1}{2}}\right\|_{\mathcal{K S}_{n-1}}\|f\|_{L^{2}} .
$$

Then, Stein's complex interpolation between (2.8) and (2.9) would give

$$
\left\|T_{1} f\right\|_{L^{2}} \leq C\left\|V^{\frac{n-1}{2}}\right\|_{\mathcal{K S}_{n-1}}^{\frac{2}{n-1}}\|f\|_{L^{2}}
$$

as desired.

It remains to show (2.9). For this, we will use the following known integral kernel $K_{\lambda}$ of $(-\Delta-z)^{-\lambda}(c f .[15,21)$ :

$$
K_{\lambda}(x)=\frac{e^{\lambda^{2}} 2^{-\lambda+1}}{(2 \pi)^{n / 2} \Gamma(\lambda) \Gamma(n / 2-\lambda)}\left(\frac{z}{|x|^{2}}\right)^{\frac{1}{2}\left(\frac{n}{2}-\lambda\right)} B_{n / 2-\lambda}\left(\sqrt{z|x|^{2}}\right),
$$


where $B_{\nu}(w)$ is the Bessel kernel of the third kind which satisfies for Re $w>0$

$$
\left|e^{\nu^{2}} \nu B_{\nu}(w)\right| \leq C|w|^{-|R e \nu|}, \quad|w| \leq 1,
$$

and

$$
\left|B_{\nu}(w)\right| \leq C_{R e \nu} e^{-\operatorname{Re} w}|w|^{-1 / 2}, \quad|w| \geq 1 .
$$

See 21, p. 339 for details. The key point is that the kernel $K_{\lambda}$ can be controlled by that of the fractional integral operator $I_{\alpha}$ which is defined for $0<\alpha<n$ by

$$
I_{\alpha} f(x)=\int_{\mathbb{R}^{n}} \frac{f(y)}{|x-y|^{n-\alpha}} d y .
$$

To show this, note first that $\operatorname{Re}\left(\sqrt{z|x|^{2}}\right)=|x| \cos \left(\frac{1}{2} \arg z\right)>0$ for $x \neq 0$, since $-\pi<\arg z \leq \pi$ by the principal branch, and $z \notin \mathbb{R}$. Then, if $\operatorname{Re}(\lambda)=(n-1) / 2$, it follows from (2.10) that for $|x| \leq 1$,

$$
\begin{aligned}
\left|K_{\lambda}(x)\right| & \leq C\left|\frac{e^{\lambda^{2}} e^{-(n / 2-\lambda)^{2}}}{n / 2-\lambda}\left(\frac{z}{|x|^{2}}\right)^{\frac{1}{2}\left(\frac{n}{2}-\lambda\right)} e^{(n / 2-\lambda)^{2}}(n / 2-\lambda) B_{n / 2-\lambda}\left(\sqrt{z|x|^{2}}\right)\right| \\
& \leq\left.\left. C\left|z^{\frac{1}{2}\left(\frac{n}{2}-\lambda\right)}\right| x\right|^{-\left(\frac{n}{2}-\lambda\right)}|| \sqrt{z|x|^{2}}\right|^{-1 / 2} \\
& \leq C e^{\operatorname{Im}(\lambda) \frac{1}{2} \arg z}|x|^{-1} \\
& \leq C e^{\frac{\pi}{2}|\operatorname{Im}(\lambda)|}|x|^{-1} .
\end{aligned}
$$

On the other hand, using (2.11), one has for $|x| \geq 1$

$$
\begin{aligned}
\left|K_{\lambda}(x)\right| & \leq C\left|\left(\frac{z}{|x|^{2}}\right)^{\frac{1}{2}\left(\frac{n}{2}-\lambda\right)} B_{n / 2-\lambda}\left(\sqrt{z|x|^{2}}\right)\right| \\
& \leq\left.\left. C\left|z^{\frac{1}{2}\left(\frac{n}{2}-\lambda\right)}\right| x\right|^{-\left(\frac{n}{2}-\lambda\right)}|| \sqrt{z|x|^{2}}\right|^{-1 / 2} \\
& \leq C e^{\frac{\pi}{2}|\operatorname{Im}(\lambda)|}|x|^{-1} .
\end{aligned}
$$

Hence for $\operatorname{Re}(\lambda)=(n-1) / 2, K_{\lambda}(x)$ is controlled by the kernel $|x|^{-1}$ of $I_{n-1}$.

Now we use the following lemma, which characterizes weighted $L^{2}$ estimates for fractional integrals, due to Kerman and Sawyer 24] (see Theorem 2.3 there and also Lemma 2.1 in [3]):

Lemma 2.4. Let $0<\alpha<n$. Assume that $w$ is a non-negative measurable function on $\mathbb{R}^{n}$. Then there exists a constant $C_{w}$ depending on $w$ such that $w \in \mathcal{K} \mathcal{S}_{\alpha}$ if and only if the two equivalent estimates

$$
\left\|I_{\alpha / 2} f\right\|_{L^{2}(w)} \leq C_{w}\|f\|_{L^{2}}
$$

and

$$
\left\|I_{\alpha / 2} f\right\|_{L^{2}} \leq C_{w}\|f\|_{L^{2}\left(w^{-1}\right)}
$$

are valid for all measurable functions $f$ on $\mathbb{R}^{n}$. Furthermore, the constant $C_{w}$ may be taken to be a constant multiple of $\|w\|_{\mathcal{K S}_{\alpha}}^{1 / 2}$.

First, we note that the two estimates in the lemma directly imply that

$$
\left\||V|^{1 / 2} I_{\alpha}\left(|V|^{1 / 2} f\right)\right\|_{L^{2}} \leq C\|V\|_{\mathcal{K} \mathcal{S}_{\alpha}}\|f\|_{L^{2}} .
$$


Then, since $\left|K_{\lambda}(x)\right| \leq C e^{\frac{\pi}{2}|\operatorname{Im}(\lambda)|}|x|^{-1}$ for $\operatorname{Re}(\lambda)=(n-1) / 2$, using (2.13) with $\alpha=n-1$, we get the desired bound (2.9). Indeed,

$$
\begin{aligned}
\left\|T_{\lambda} f\right\|_{L^{2}} & =\left\|\left|V^{\frac{n-1}{2}}\right|^{1 / 2}(-\Delta-z)^{-\lambda}\left[\left|V^{\frac{n-1}{2}}\right|^{1 / 2}\left(|V|^{i \operatorname{Im}(\lambda) / 2} f\right)\right]\right\|_{L^{2}} \\
& \leq C e^{\frac{\pi}{2}|\operatorname{Im}(\lambda)|}\left\|\left|V^{\frac{n-1}{2}}\right|^{1 / 2} I_{n-1}\left[\left|V^{\frac{n-1}{2}}\right|^{1 / 2}\left(|V|^{i \operatorname{Im}(\lambda) / 2} f\right)\right]\right\|_{L^{2}} \\
& \leq C e^{\frac{\pi}{2}|\operatorname{Im}(\lambda)|}\left\|V^{\frac{n-1}{2}}\right\|_{\mathcal{K} \mathcal{S}_{n-1}}\|f\|_{L^{2}} .
\end{aligned}
$$

This completes the proof.

\section{LIMITING ABSORPTION PRINCIPLE AND FOURIER RESTRICTION ESTIMATES}

In this section we study the limiting absorption principle in spectral theory and its relation with weighted restriction estimates in harmonic analysis. This relationship seems to be well known, but to the best of our knowledge, it was treated in the literature $([3,4])$ only in certain particular cases. Here we put it in a more abstract framework for our purpose in this paper. The resulting restriction estimates will be fundamentally used in the next section for obtaining weighted $L^{2}$ estimates for the Schrödinger propagator. Alternatively, we will obtain them more directly by appealing to Kato $H$-smoothing theory.

First we recall some basic notions and facts from spectral theory. Let $T$ be a closed ${ }^{3}$ linear operator on a Hilbert space $\mathcal{H}$ over $\mathbb{C}$. Then we denote by $\rho(T)$ the resolvent set of $T$ which is the set of $z \in \mathbb{C}$ for which $T-z$ is invertible and the inverse $(T-z)^{-1}$ is a bounded operator on $\mathcal{H}$. The spectrum $\sigma(T)=\mathbb{C} \backslash \rho(T)$ is given by the complement of the resolvent set. One of the most fundamental facts is that the resolvent $R_{T}(z):=(T-z)^{-1}$ is an analytic operator-valued function on $\rho(T)$ and the operator norm $\left\|R_{T}(z)\right\|$ satisfies

$$
\left\|R_{T}(z)\right\| \geq[\operatorname{dist}(z, \sigma(T))]^{-1} .
$$

(See, for example, 42.) The point here is that the norm of $R_{T}(z)$ as a map from $\mathcal{H}$ to $\mathcal{H}$ diverges as $z$ approaches $\sigma(T)$. But there is a principle that $R_{T}(z)$ can remain bounded in a sense. This is referred to as the limiting absorption principle.

Of special interest is the free resolvent which is usually denoted by $R_{0}(z)=$ $(-\Delta-z)^{-1}$ on $L^{2}\left(\mathbb{R}^{n}\right)$. In this case, the spectrum is $[0, \infty)$ and there is a classical result due to Agmon [1] which states that the limits

$$
\lim _{\varepsilon \rightarrow 0} R_{0}(\lambda \pm i \varepsilon):=R_{0}(\lambda \pm i 0)
$$

exist for $\lambda \in(0, \infty)$ in the norm of bounded operators from $L^{2}\left(\langle x\rangle^{s} d x\right)$ to $L^{2}\left(\langle x\rangle^{-s} d x\right)$ for $s>1$. (Here $\langle x\rangle=\left(1+|x|^{2}\right)^{1 / 2}$.) This shows that the free resolvent can remain bounded between weighted $L^{2}$ spaces as $z$ approaches the spectrum (except $0)$. Furthermore, the principle holds in the following weak form which is called the weak limiting absorption principle: For $f, g \in L^{2}\left(\langle x\rangle^{-s} d x\right)$

$$
\lim _{\varepsilon \rightarrow 0}\left\langle R_{0}(\lambda \pm i \varepsilon) f, g\right\rangle=\left\langle R_{0}(\lambda \pm i 0) f, g\right\rangle,
$$

where $\langle$,$\rangle denotes the usual inner product on the Hilbert space L^{2}\left(\mathbb{R}^{n}\right)$. These principles play a key role in the study of Schrödinger operators as well as Helmholtz equations.

\footnotetext{
${ }^{3}$ If $T$ is not closed, then $T-z$ and $(T-z)^{-1}$ are not closed. So, $\rho(T)$ is empty. This is why $T$ is assumed to be closed.
} 
For our purpose, it is enough to consider the following weak principle:

Proposition 3.1. Let $n \geq 2$ and let $V: \mathbb{R}^{n} \rightarrow \mathbb{C}$ be such that the resolvent estimate

$$
\left\|R_{0}(z) f\right\|_{L^{2}(|V|)} \leq C(V)\|f\|_{L^{2}\left(|V|^{-1}\right)}
$$

holds uniformly in $z \in \mathbb{C} \backslash \mathbb{R}$. Then, for $\lambda>0$ there exists the weak limit

$$
\lim _{\varepsilon \rightarrow 0}\left\langle R_{0}(\lambda \pm i \varepsilon) f, g\right\rangle:=\left\langle R_{0}(\lambda \pm i 0) f, g\right\rangle
$$

whenever $f, g \in L^{2}\left(|V|^{-1}\right)$. Furthermore, $R_{0}(\lambda \pm i 0)$ satisfies

$$
\left\|R_{0}(\lambda \pm i 0) f\right\|_{L^{2}(|V|)} \leq C(V)\|f\|_{L^{2}\left(|V|^{-1}\right)}
$$

and can be given by the following distributional identity:

$$
R_{0}(\lambda \pm i 0) f(x)= \pm \frac{i \pi}{2 \sqrt{\lambda}} \widehat{d \sigma \sqrt{\lambda}} * f(x)+p \cdot v \cdot \int_{\mathbb{R}^{n}} e^{i x \cdot \xi} \frac{\widehat{f}(\xi)}{|\xi|^{2}-\lambda} d \xi .
$$

Here, d $\sigma_{r}$ denotes the induced Lebesgue measure on the sphere $S_{r}^{n-1}$ in $\mathbb{R}^{n}$ with radius $r$, and the singular integral in (3.4) is taken in the principal value sense.

Assuming for the moment this proposition, we first explain how to deduce Fourier restriction estimates from the weak principle. From (3.3) and taking the imaginary part of the operator $R_{0}(\lambda \pm i 0)$ in (3.4) , it follows that

$$
\left\|\widehat{d \sigma_{r}} * f\right\|_{L^{2}(|V|)} \leq r C(V)\|f\|_{L^{2}\left(|V|^{-1}\right)} .
$$

Then, using this and the standard $T T^{*}$ argument of Tomas [43, we see that

$$
\begin{aligned}
\int_{S_{r}^{n-1}}|\widehat{f}|^{2} d \sigma=\int_{S_{r}^{n-1}} \widehat{f} \bar{f} d \sigma & =\int_{\mathbb{R}^{n}} f\left(f * \widehat{d \sigma_{r}}\right) d x \\
& \leq\|f\|_{L^{2}\left(|V|^{-1}\right)}\left\|f * \widehat{d \sigma_{r}}\right\|_{L^{2}(|V|)} \\
& \leq r C(V)\|f\|_{L^{2}\left(|V|^{-1}\right)}^{2}
\end{aligned}
$$

Namely, we get the weighted $L^{2}$ restriction estimate

$$
\|\widehat{f}\|_{L^{2}\left(S_{r}^{n-1}\right)} \leq r^{1 / 2} C(V)^{1 / 2}\|f\|_{L^{2}\left(|V|^{-1}\right)} .
$$

By duality this is equivalent to

$$
\left\|\widehat{f d \sigma_{r}}\right\|_{L^{2}(|V|)} \leq r^{1 / 2} C(V)^{1 / 2}\|f\|_{L^{2}\left(S_{r}^{n-1}\right)} .
$$

The first estimate (3.5) can also be deduced immediately from these two equivalent estimates, and what we have just explained is summarized in the following corollary.

Corollary 3.2. Let $n \geq 2$ and let $V: \mathbb{R}^{n} \rightarrow \mathbb{C}$ be such that the resolvent estimate (3.1) holds. Then the following three equivalent estimates hold:

$$
\begin{gathered}
\left\|\widehat{d \sigma_{r}} * f\right\|_{L^{2}(|V|)} \leq r C(V)\|f\|_{L^{2}\left(|V|^{-1}\right)}, \\
\|\widehat{f}\|_{L^{2}\left(S_{r}^{n-1}\right)} \leq r^{1 / 2} C(V)^{1 / 2}\|f\|_{L^{2}\left(|V|^{-1}\right)}, \\
\left\|\widehat{f d \sigma_{r}}\right\|_{L^{2}(|V|)} \leq r^{1 / 2} C(V)^{1 / 2}\|f\|_{L^{2}\left(S_{r}^{n-1}\right)} .
\end{gathered}
$$

Remark 3.3. Given a class $\mathcal{R}$ of resolvent type, this corollary clearly holds for $V \in \mathcal{R}$ with $C(V) \sim[V]_{\mathcal{R}}$. In the cases of $\mathcal{R}=\mathcal{L}^{2, p}, p>(n-1) / 2$, and $\mathcal{S}_{3}$, the above restriction estimates can be found in [8] and [3], respectively. In view of the resolvent estimates in Theorem 2.2 , these previous results are now extended to the class $\mathcal{S}_{n}$. 
Proof of Proposition 3.1. We basically follow the argument of Agmon 11. In fact the argument given by him was for the case $V(x)=\langle x\rangle^{-s}, s>1$, but it goes through in our abstract setting.

Let us first consider the analytic function $F(z)=\left\langle R_{0}(z) f, g\right\rangle$ for $z \in \mathbb{C} \backslash \mathbb{R}$. Then it follows from (3.1) that

$$
\left|\left\langle R_{0}(z) f, g\right\rangle\right| \leq C(V)\|f\|_{L^{2}\left(|V|^{-1}\right)}\|g\|_{L^{2}\left(|V|^{-1}\right)} .
$$

Since this estimate is uniform in $z$ and $C_{0}^{\infty}$ is dense in $L^{2}\left(|V|^{-1}\right)$, by a standard limiting argument, it suffices to show (3.2) for $f, g \in C_{0}^{\infty}$. Here, to see that $C_{0}^{\infty}$ is dense in $L^{2}\left(|V|^{-1}\right)$, first consider $D_{n}=\left\{x \in \mathbb{R}^{n}:|V|^{-1} \geq 1 / n\right\}$. Then, for $f \in$ $L^{2}\left(|V|^{-1}\right)$ the function $\chi_{D_{n}} f$ is contained in $L^{2} \cap L^{2}\left(|V|^{-1}\right)$, and $\chi_{D_{n}} f|V|^{-1 / 2} \rightarrow$ $f|V|^{-1 / 2}$ as $n \rightarrow \infty$. By the Lebesgue dominated convergence theorem, it now follows that $\chi_{D_{n}} f|V|^{-1 / 2} \rightarrow f|V|^{-1 / 2}$ in $L^{2}$. This means that $L^{2} \cap L^{2}\left(|V|^{-1}\right)$ is dense in $L^{2}\left(|V|^{-1}\right)$. On the other hand, it is easy to see that $C_{0}^{\infty}$ is dense in $L^{2} \cap L^{2}\left(|V|^{-1}\right)$ with respect to the norm $L^{2}\left(|V|^{-1}\right)$ using a $C_{0}^{\infty}$ approximate identity. Consequently, $C_{0}^{\infty}$ is dense in $L^{2}\left(|V|^{-1}\right)$.

Now, using Parseval's formula and changing to polar coordinates, we see that

$$
\begin{aligned}
\left\langle R_{0}(z) f, g\right\rangle & =\int_{\mathbb{R}^{n}} \frac{\widehat{f}(\xi) \overline{\widehat{g}(\xi)}}{|\xi|^{2}-z} d \xi \\
& =\frac{1}{2} \int_{0}^{\infty} \frac{t^{(n-2) / 2}}{t-z}\left(\int_{|\omega|=1} \widehat{f}(\sqrt{t} \omega) \overline{\widehat{g}(\sqrt{t} \omega)} d \omega\right) d t
\end{aligned}
$$

Thus, by a well-known continuity property of Cauchy type integrals (the SokhotskyPlemelj formula), $\left\langle R_{0}(z) f, g\right\rangle$ has continuous boundary values given by

$$
\lim _{z \rightarrow \lambda, \pm I m z>0}\left\langle R_{0}(z) f, g\right\rangle= \pm \frac{\pi i}{2 \sqrt{\lambda}} \int_{|\xi|=\sqrt{\lambda}} \widehat{f}(\xi) \overline{\widehat{g}(\xi)} d \sigma+p . v \cdot \int_{\mathbb{R}^{n}} \frac{\widehat{f}(\xi) \overline{\widehat{g}(\xi)}}{|\xi|^{2}-\lambda} d \xi
$$

on both sides of $(0, \infty)$ (see (4.7) in [1]). This means that for $f \in L^{2}\left(|V|^{-1}\right)$ there exists the weak limit

$$
w-\lim _{z \rightarrow \lambda, \pm \operatorname{Im} z>0} R_{0}(z) f:=R_{0}(\lambda \pm i 0) f
$$

in $L^{2}(|V|)$. Namely, for $f, g \in L^{2}\left(|V|^{-1}\right)$,

$$
\lim _{z \rightarrow \lambda, \pm \operatorname{Im} z>0}\left\langle R_{0}(z) f, g\right\rangle=\left\langle R_{0}(\lambda \pm i 0) f, g\right\rangle,
$$

and the identity (3.4) follows immediately from (3.7). Now, by duality the estimate (3.3) is deduced from (3.2) and (3.1). Indeed, note that

$$
\begin{aligned}
\left\|R_{0}(\lambda \pm i 0) f\right\|_{L^{2}(|V|)} & =\sup _{\|\widetilde{g}\|_{L^{2}} \leq 1}\left|\left\langle|V|^{1 / 2} R_{0}(\lambda \pm i 0) f, \widetilde{g}\right\rangle\right| \\
& =\sup _{\|\widetilde{g}\|_{L^{2}} \leq 1}\left|\left\langle R_{0}(\lambda \pm i 0) f,|V|^{1 / 2} \widetilde{g}\right\rangle\right| \\
& =\sup _{\|g\|_{L^{2}\left(|V|^{-1}\right)} \leq 1}\left|\left\langle R_{0}(\lambda \pm i 0) f, g\right\rangle\right|,
\end{aligned}
$$

where $g=|V|^{1 / 2} \widetilde{g}$. By (3.2) and (3.1), this readily leads to

$$
\left\|R_{0}(\lambda \pm i 0) f\right\|_{L^{2}(|V|)} \leq C(V)\|f\|_{L^{2}\left(|V|^{-1}\right)} .
$$

This completes the proof. 


\section{Weighted $L^{2}$ estimates for the Schrödinger propagator}

Let us first consider the following initial value problem for the free Schrödinger equation:

$$
\left\{\begin{array}{l}
i \partial_{t} u+\Delta u=0 \\
u(x, 0)=u_{0}(x)
\end{array}\right.
$$

In view of the Fourier transform, the solution is explicitly given by

$$
u(x, t)=e^{i t \Delta} u_{0}(x)=(2 \pi)^{-n} \int_{\mathbb{R}^{n}} e^{i x \cdot \xi} e^{-i t|\xi|^{2}} \widehat{u_{0}}(\xi) d \xi,
$$

where the evolution operator $e^{i t \Delta}$ is called the free Schrödinger propagator. Then the upshot of this section is the following weighted $L^{2}$ estimate for the propagator, which will play a key role in the next section in obtaining our abstract Carleman estimates.

Proposition 4.1. Let $n \geq 2$ and let $V: \mathbb{R}^{n} \rightarrow \mathbb{C}$ be such that the resolvent estimate

$$
\left\|R_{0}(z) f\right\|_{L^{2}(|V|)} \leq C(V)\|f\|_{L^{2}\left(|V|^{-1}\right)}
$$

holds uniformly in $z \in \mathbb{C} \backslash \mathbb{R}$. Then we have

$$
\left\|\int_{-\infty}^{\infty} e^{i(t-s) \Delta} F(\cdot, s) d s\right\|_{L_{t, x}^{2}(|V|)} \leq C C(V)\|F\|_{L_{t, x}^{2}\left(|V|^{-1}\right)},
$$

where $F: \mathbb{R}^{n+1} \rightarrow \mathbb{C}$ is a function in $L_{t, x}^{2}\left(|V|^{-1}\right)$.

The basic approach for this is to obtain the estimate

$$
\left\|e^{i t \Delta} f\right\|_{L_{t, x}^{2}(|V|)} \leq C C(V)^{1 / 2}\|f\|_{2},
$$

which may be referred to as the weighted $L^{2}$ homogeneous Strichartz estimate. (In Section 8 we will also obtain the corresponding inhomogeneous estimate to study the well-posedness of the Schrödinger equation.) Indeed, by duality this is equivalent to

$$
\left\|\int_{-\infty}^{\infty} e^{-i s \Delta} F(\cdot, s) d s\right\|_{2} \leq C C(V)^{1 / 2}\|F\|_{L_{t, x}^{2}\left(|V|^{-1}\right)},
$$

and so one gets (4.3) by combining (4.4) and (4.5).

In this regard, we aim at proving the following lemma. We shall give two proofs of that. The first is a concrete one using the weighted $L^{2}$ restriction estimate given in the previous section. On the other hand, the second is a more direct one appealing to Kato $H$-smoothing theory.

Lemma 4.2. Let $n \geq 2$ and let $V: \mathbb{R}^{n} \rightarrow \mathbb{C}$ be such that the resolvent estimate (4.2) holds. Then we have

$$
\left\|e^{i t \Delta} f\right\|_{L_{t, x}^{2}(|V|)} \leq C C(V)^{1 / 2}\|f\|_{2} .
$$

\footnotetext{
${ }^{4}$ Consider the operator $T f(x, t)=|V(x)|^{1 / 2} e^{i t \Delta} f(x)$. Then, $\|T f\|_{L_{t, x}^{2}} \leq C C(V)^{1 / 2}\|f\|_{2}$ from (4.4). Since the adjoint operator of $T$ is now given as $T^{*} G(x)=\int_{-\infty}^{\infty} e^{-i s \Delta}\left(|V(\cdot)|^{1 / 2} G(\cdot, s)\right) d s$, by duality, $\left\|T^{*} G\right\|_{2} \leq C C(V)^{1 / 2}\|G\|_{L_{t, x}^{2}}$, which is equivalent to (4.5).
} 
Remark 4.3. The estimate (4.6) was first obtained implicitly in 34 for FeffermanPhong potentials $V \in \mathcal{L}^{2, p}, p>(n-1) / 2, n \geq 3$, and this was extended in 3 to the Kerman-Sawyer class $\mathcal{K}_{2}$ only for $n=3$. By combining the resolvent estimates in Theorem 2.2 and this lemma, we can extend these previous results to the class $\mathcal{S}_{n}$

Proof of Lemma 4.2. The proof is quite standard and based on the weighted $L^{2}$ restriction estimate given in the previous section. Indeed, using polar coordinates and changing variables $r^{2}=\lambda$, one can see that

$$
\begin{aligned}
e^{i t \Delta} f & =\int_{0}^{\infty} e^{-i t r^{2}} \int_{S_{r}^{n-1}} e^{i x \cdot \xi} \widehat{f}(\xi) d \sigma_{r}(\xi) d r \\
& =\frac{1}{2} \int_{0}^{\infty} e^{-i t \lambda} \int_{S_{\sqrt{\lambda}}^{n-1}} e^{i x \cdot \xi} \widehat{f}(\xi) d \sigma_{\sqrt{\lambda}}(\xi) \lambda^{-1 / 2} d \lambda .
\end{aligned}
$$

By Plancherel's theorem in $t$, it now follows that

$$
\begin{aligned}
\left\|e^{i t \Delta} f\right\|_{L_{t, x}^{2}(|V|)}^{2} & \leq C \int_{\mathbb{R}^{n}}\left(\int_{0}^{\infty}\left|\int_{S_{\sqrt{\lambda}}^{n-1}} e^{i x \cdot \xi} \widehat{f}(\xi) d \sigma_{\sqrt{\lambda}}(\xi)\right|^{2} \lambda^{-1} d \lambda\right)|V(x)| d x \\
& \leq C \int_{0}^{\infty}\left(\int_{\mathbb{R}^{n}}\left|\int_{S_{r}^{n-1}} e^{i x \cdot \xi} \widehat{f}(\xi) d \sigma_{r}(\xi)\right|^{2}|V(x)| d x\right) r^{-1} d r .
\end{aligned}
$$

Combining this and the estimate (3.6) in Corollary 3.2, one gets

$$
\begin{aligned}
\left\|e^{i t \Delta} f\right\|_{L_{t, x}^{2}(|V|)}^{2} & \leq C C(V) \int_{0}^{\infty} \int_{S_{r}^{n-1}}|\widehat{f}(\xi)|^{2} d \sigma_{r}(\xi) d r \\
& =C C(V)\|f\|_{2}^{2}
\end{aligned}
$$

as desired.

Now we derive (4.6) more directly from the resolvent estimate appealing to Kato $H$-smoothing theory. The notion of $H$-smoothing due to Kato [18 first appeared in the context of scattering theory, and its usefulness for dispersive equations was revealed in some recent works $([11,32])$. We shall use a version of the notion to suit our purpose.

Let $H$ be a self-adjoint operator in a Hilbert space $\mathcal{H}$ whose resolvent is $R_{H}(z)=$ $(H-z)^{-1}$ for $z \in \mathbb{C} \backslash \mathbb{R}$. Let $T$ be a densely defined, closed operator on $\mathcal{H}$ with domain $D(T)$. Then the following lemma due to Kato [18, (see also [31, XIII.7) allows us to employ the resolvent estimate for $H$ to obtain a space-time estimate for the Schrödinger propagator.

Lemma 4.4. Assume that there exists a constant $\widetilde{C}>0$ such that for $g \in D\left(T^{*}\right)$,

$$
\sup _{z \in \mathbb{C} \backslash \mathbb{R}}\left\|T R_{H}(z) T^{*} g\right\|_{\mathcal{H}} \leq \widetilde{C}\left\|_{g}\right\|_{\mathcal{H}}
$$

Then the operator $T$ is $H$-smooth, i.e., $e^{i t H} f \in D(T)$ for $f \in \mathcal{H}$ and almost every $t$, and

$$
\int_{\mathbb{R}}\left\|T e^{i t H} f\right\|_{\mathcal{H}}^{2} d t \leq \widetilde{C}\|f\|_{\mathcal{H}}^{2}
$$


Alternate proof of Lemma 4.2. Now we appeal to this lemma in order to prove Lemma 4.2. By replacing $f=|V|^{1 / 2} g$ in the resolvent estimate (4.2) we see that

$$
\sup _{z \in \mathbb{C} \backslash \mathbb{R}}\left\||V|^{1 / 2} R_{0}(z)|V|^{1 / 2} g\right\|_{L^{2}} \leq C(V)\|g\|_{L^{2}} .
$$

Hence, applying Lemma 4.4 with $H=-\Delta, \mathcal{H}=L^{2}$, and $T: f \mapsto|V|^{1 / 2} f$, we immediately get the desired estimate (4.6). In doing so, it seems to be more or less complicated to check that the multiplication operator $T: f \mapsto|V|^{1 / 2} f$ is a densely defined, closed operator on $L^{2}$ with $D(T)=\left\{f \in L^{2}: T f \in L^{2}\right\}$. Let us now check this. First, it is easy to see that $D(T)$ is dense in $L^{2}$. In fact, consider $D_{n}=\left\{x \in \mathbb{R}^{n}:|V|^{1 / 2} \leq n\right\}$. Then, for $f \in L^{2}$ the function $\chi_{D_{n}} f$ is contained in $D(T)$, and $\chi_{D_{n}} f \rightarrow f$ as $n \rightarrow \infty$. By the Lebesgue dominated convergence theorem, it now follows that $\chi_{D_{n}} f \rightarrow f$ in $L^{2}$. So, $D(T)$ is dense in $L^{2}$. Next, note that since $|V|^{1 / 2}$ is real, $T$ is trivially self-adjoint, i.e., $T=T^{*}$, and so $T^{*}$ is also dense in $L^{2}$. From these facts, $T$ is closable and its closure can be given by $\bar{T}=T^{* *}$. (See, for example, Theorem VIII.1 in [29].) Thus, $\bar{T}=T^{* *}=T^{*}=T$. That is, $T$ is closed.

\section{Carleman estimates}

This section is devoted to proving the following abstract Carleman estimate which means that a potential for the resolvent estimate can be made to work for the Carleman estimate in an abstract way.

Proposition 5.1. Let $n \geq 2$ and let $[V]$ be the least constant $C(V)$ for which the resolvent estimate

$$
\left\|R_{0}(z) f\right\|_{L^{2}(|V|)} \leq C(V)\|f\|_{L^{2}\left(|V|^{-1}\right)}
$$

holds uniformly in $z \in \mathbb{C} \backslash \mathbb{R}$. Then we have for $u \in C_{0}^{\infty}\left(\mathbb{R}^{n+1}\right)$

$$
\left\|e^{\beta\langle(x, t), \nu\rangle} u\right\|_{L_{t, x}^{2}(|V(x)|)} \leq C[V]\left\|e^{\beta\langle(x, t), \nu\rangle}\left(i \partial_{t}+\Delta\right) u\right\|_{L_{t, x}^{2}\left(|V(x)|^{-1}\right)}
$$

with a constant $C$ independent of $\beta \in \mathbb{R}$ and $\nu \in \mathbb{R}^{n+1}$, if $|V| \in A_{2}\left(\nu^{\prime}\right)$. Here, recall that $\nu^{\prime}=\left(\nu_{1}, \ldots, \nu_{n}\right)$ for $\nu=\left(\nu_{1}, \ldots, \nu_{n}, \nu_{n+1}\right)$.

Remark 5.2. The assumption $|V| \in A_{2}\left(\nu^{\prime}\right)$ is the only one needed in order to derive (5.2) from (5.1). Once a suitable class $\mathcal{R}$ is established, (5.2) holds for $V \in \mathcal{R}$ with $[V]$ replaced by $[V]_{\mathcal{R}}$. In this concrete setting there are some classes of $\mathcal{R}$ where the $A_{2}$ assumption is superfluous. See a comment below the proof of Proposition 5.3 for details.

To obtain (5.2), we first convert it into the following form which seems at first glance to be easier to handle:

$$
\|u\|_{L_{t, x}^{2}(|V(x)|)} \leq C[V]\left\|\left(i \partial_{t}+\Delta+P(D)\right) u\right\|_{L_{t, x}^{2}\left(|V(x)|^{-1}\right)},
$$

where $P(D)=-2 \beta\left\langle\nu^{\prime}, \nabla\right\rangle+\beta^{2}\left|\nu^{\prime}\right|^{2}-i \beta \nu_{n+1}$ arises from a computation of the conjugated operator $e^{\beta\langle(x, t), \nu\rangle}\left(i \partial_{t}+\Delta\right) e^{-\beta\langle(x, t), \nu\rangle}$. Note that $e^{\beta\langle(x, t), \nu\rangle}$ no longer appears explicitly in this form although it affects $P(D)$. The key point is that $P(D)$ can be controlled uniformly in $\beta, \nu$ by decomposing $\left(i \partial_{t}+\Delta+P(D)\right)^{-1}$ in the phase space, along with the corresponding direction of the vector $\nu^{\prime}$, into a number of localized pieces which can be made in a sensible way to behave like the resolvent $(-\Delta-z)^{-1}$. These pieces will then be estimated and recombined 
successfully on weighted $L^{2}$ spaces. The $A_{2}$ assumption comes into play at this step.

In fact we obtain the Sobolev type inequality (5.3) in the following more general setting:

Proposition 5.3. Let $n \geq 2$ and let $P(D)$ be a first-order differential operator given by $P(D)=\langle\vec{c}, \nabla\rangle+z$, where $\vec{c}=\vec{a}+i \vec{b} \in \mathbb{C}^{n}$ with $\vec{a}, \vec{b} \in \mathbb{R}^{n}$, and $z \in \mathbb{C}$. Then we have for $u \in C_{0}^{\infty}\left(\mathbb{R}^{n+1}\right)$

$$
\|u\|_{L_{t, x}^{2}(|V(x)|)} \leq C[V]\left\|\left(i \partial_{t}+\Delta+P(D)\right) u\right\|_{L_{t, x}^{2}\left(|V(x)|^{-1}\right)}
$$

with a constant $C$ independent of $\vec{c}$ and $z$, if $|V| \in A_{2}(\vec{a})$.

It had been conjectured that the following estimate holds for some $p \neq 2$ :

$$
\|u\|_{L_{t, x}^{p}} \leq C\left\|\left(i \partial_{t}+\Delta+i\right) u\right\|_{L_{t, x}^{p}} .
$$

Note that the multiplier $1 /\left(-\tau-|\xi|^{2}+i\right)$ associated with $\left(i \partial_{t}+\Delta+i\right)^{-1}$ is bounded, and so (5.5) is trivially satisfied for $p=2$ by Plancherel's theorem. Interest in (5.5) came from the work of Calderón [5] concerning the $L^{p}$ boundedness of Fourier multipliers given by bounded rational functions, and it was shown in 23 , that the conjecture is false, namely, (5.5) holds only for $p=2$. Our estimate (5.4) can be viewed as a weighted $L^{2}$ version, with the general first-order term $P(D)$, of (5.5).

Proof of Proposition [5.3. First, note that $[V]$ is invariant under translations $T$ and rotations $R$. That is, $[V(T x)]=[V]$ and $[V(R x)]=[V]$. It is an elementary matter to check this. Of course, $[C V(x)]=C[V]$ for constants $C>0$. Then by elementary arguments, the inequality (5.4) can be reduced to the following particular case where $P(D)=\partial_{x_{n}}$ :

$$
\|u\|_{L_{t, x}^{2}(|V|)} \leq C[V]\left\|\left(i \partial_{t}+\Delta+\partial_{x_{n}}\right) u\right\|_{L_{t, x}^{2}\left(|V|^{-1}\right)} .
$$

In fact, by setting $u=e^{-i\langle\vec{b} / 2, x\rangle} v, \vec{b} \in \mathbb{R}^{n}$, it follows that

$$
\left(i \partial_{t}+\Delta+P(D)\right) u=e^{-i\langle\vec{b} / 2, x\rangle}\left(i \partial_{t}+\Delta+\langle\vec{c}-i \vec{b}, \nabla\rangle+z-i\langle\vec{c}, \vec{b} / 2\rangle-|\vec{b} / 2|^{2}\right) v .
$$

Hence one may assume that $\vec{c} \in \mathbb{R}^{n}$. Similarly, for $z=a+i b \in \mathbb{C}$, by setting $u=e^{i a t} v$, one can see that

$$
\left(i \partial_{t}+\Delta+P(D)\right) u=e^{i a t}\left(i \partial_{t}+\Delta+\langle\vec{c}, \nabla\rangle+i b\right) v .
$$

From this one may also assume that $\operatorname{Re} z=0$. Since the Laplacian $\Delta$ is trivially invariant under rotations, and so is $[V]$, by a simple rotation argument the assumption $|V| \in A_{2}(\vec{a})$ is reduced to the case where $|V| \in A_{2}(\mathbb{R})$ in the $x_{n}$ variable uniformly in other variables, and $\langle\vec{c}, \nabla\rangle=c \partial_{x_{n}}$ with $c \in \mathbb{R}$. So far, we have explained how to reduce the matter to the case where $P(D)=c \partial / \partial x_{n}+i b$ with $c, b \in \mathbb{R}$. For simplicity of notation, we shall also assume that $c=1$ and $b=0$, because it does not affect all the arguments in the proof.

Our basic plan for (5.6) is to decompose the inverse $\left(i \partial_{t}+\Delta+\partial_{x_{n}}\right)^{-1}$ in the Fourier transform side, along with the $\xi_{n}$ axis, into a number of localized pieces which can be made to behave like the resolvent $(-\Delta-z)^{-1}$, and to estimate and recombine them on weighted $L^{2}$ spaces using Proposition 4.1 and appealing to real variable theory in 25$]$. 
Let us now rewrite (5.6) in the Fourier transform side as

$$
\left\|\mathcal{F}^{-1}\left(\frac{\widehat{f}(\xi, \tau)}{\tau+|\xi|^{2}+i \xi_{n}}\right)\right\|_{L_{t, x}^{2}(|V|)} \leq C[V]\|f\|_{L_{t, x}^{2}\left(|V|^{-1}\right)}
$$

for $f \in C_{0}^{\infty}\left(\mathbb{R}^{n+1}\right)$. Setting $m(\xi, \tau)=\left(\tau+|\xi|^{2}+i \xi_{n}\right)^{-1}$, we define the multiplier operator $\mathcal{T}$ by

$$
\widehat{\mathcal{T} f}(\xi, \tau)=m(\xi, \tau) \widehat{f}(\xi, \tau) .
$$

For $k \in \mathbb{Z}$, we also set $\phi_{k}(r)=\phi\left(2^{k} r\right)$ for $\phi \in C_{0}^{\infty}(\mathbb{R})$ which is such that $\phi(r)=1$ if $|r| \sim 1$, and $\phi(r)=0$ otherwise. Using this, we decompose $\mathcal{T}$ into the localized operators $\mathcal{T}_{k}, k \in \mathbb{Z}$, which are given by

$$
\widehat{\mathcal{T}_{k} f}(\xi, \tau)=m(\xi, \tau) \chi_{k}\left(\xi_{n}\right) \widehat{f}(\xi, \tau) .
$$

Now, (5.7) becomes equivalent to

$$
\left\|\sum_{k \in \mathbb{Z}} \mathcal{T}_{k} f\right\|_{L_{t, x}^{2}(|V|)} \leq C[V]\|f\|_{L_{t, x}^{2}\left(|V|^{-1}\right)} .
$$

To show this, we assume for the moment that

$$
\left\|\mathcal{T}_{k} f\right\|_{L_{t, x}^{2}(|V|)} \leq C[V]\|f\|_{L_{t, x}^{2}\left(|V|^{-1}\right)}
$$

with $C$ independent of $k \in \mathbb{Z}$. Then, by the Littlewood-Paley theorem on weighted $L^{2}$ spaces with weights in the $A_{2}$ class (see Theorem 1 in 25]), we can get the desired estimate (5.8). Indeed, since $|V| \in A_{2}(\mathbb{R})$ in the $x_{n}$ variable uniformly in other variables, by the Littlewood-Paley theorem in $x_{n}$ and using (5.9) it follows that

$$
\begin{aligned}
\left\|\sum_{k} \mathcal{T}_{k} f\right\|_{L_{t, x}^{2}(|V|)} & \leq C\left\|\left(\sum_{k \in \mathbb{Z}}\left|\mathcal{T}_{k} f\right|^{2}\right)^{1 / 2}\right\| \|_{L_{t, x}^{2}(|V|)} \\
& =C\left(\iint \sum_{k \in \mathbb{Z}}\left|\mathcal{T}_{k} f\right|^{2}|V| d x d t\right)^{1 / 2} \\
& =C\left(\sum_{k \in \mathbb{Z}}\left\|\mathcal{T}_{k} f\right\|_{L_{t, x}^{2}(|V|)}^{2}\right)^{1 / 2} \\
& \leq C[V]\left(\sum_{k \in \mathbb{Z}}\left\|f_{k}\right\|_{L_{t, x}^{2}\left(|V|^{-1}\right)}^{2}\right)^{1 / 2}
\end{aligned}
$$

where $\widehat{f}_{k}(\xi, \tau)=\chi_{k}\left(\xi_{n}\right) \widehat{f}(\xi, \tau)$. On the other hand, since $|V| \in A_{2}(\mathbb{R})$ if and only if $|V|^{-1} \in A_{2}(\mathbb{R})$, by the Littlewood-Paley theorem again, we see that

$$
\begin{aligned}
\left(\sum_{k \in \mathbb{Z}}\left\|f_{k}\right\|_{L_{t, x}^{2}\left(|V|^{-1}\right)}^{2}\right)^{1 / 2} & =\left(\iint \sum_{k \in \mathbb{Z}}\left|f_{k}\right|^{2}|V|^{-1} d x d t\right)^{1 / 2} \\
& =\left\|\left(\sum_{k \in \mathbb{Z}}\left|f_{k}\right|^{2}\right)^{1 / 2}\right\|_{L_{t, x}^{2}\left(|V|^{-1}\right)} \\
& \leq C\|f\|_{L_{t, x}^{2}\left(|V|^{-1}\right)}
\end{aligned}
$$

Combining (5.10) and (5.11), we get the desired estimate (5.8).

Now it remains to show the estimate (5.9). Equivalently, we have to show that (5.7) holds for $f \in C_{0}^{\infty}\left(\mathbb{R}^{n+1}\right)$ satisfying supp $\widehat{f} \subset\left\{(\xi, \tau) \in \mathbb{R}^{n+1}:\left|\xi_{n}\right| \sim 2^{-k}\right\}$, 
with the constant $C$ independent of $k \in \mathbb{Z}$. To do so, we first derive the following bound from the resolvent estimate:

$$
\left\|\mathcal{F}^{-1}\left(\frac{\widehat{f}(\xi, \tau)}{\tau+|\xi|^{2}+i 2^{-k}}\right)\right\|_{L_{t, x}^{2}(|V|)} \leq[V]\|f\|_{L_{t, x}^{2}\left(|V|^{-1}\right)} .
$$

In fact, note that

$$
\begin{aligned}
\left\|\mathcal{F}^{-1}\left(\frac{\widehat{f}(\xi, \tau)}{\tau+|\xi|^{2}+i 2^{-k}}\right)\right\|_{L_{t, x}^{2}(|V|)}^{2} & \\
& =\int|V| \int\left|\int e^{i t \tau}\left(\int e^{i x \cdot \xi} \frac{\widehat{f}(\xi, \tau)}{\tau+|\xi|^{2}+i 2^{-k}} d \xi\right) d \tau\right|^{2} d t d x \\
& =\int|V| \int\left|\int e^{i x \cdot \xi} \frac{\widehat{f}(\xi, \tau)}{\tau+|\xi|^{2}+i 2^{-k}} d \xi\right|^{2} d \tau d x \\
& =\iint\left|\int e^{i x \cdot \xi} \frac{\widehat{f}(\xi, \tau)}{\tau+|\xi|^{2}+i 2^{-k}} d \xi\right|^{2}|V| d x d \tau .
\end{aligned}
$$

Here we used Plancherel's theorem in $t$ for the second equality. Applying the resolvent estimate (5.1) with $z=-\tau-i 2^{-k}$ to the last term in the above and using Plancherel's theorem in $\tau$, we get

$$
\begin{aligned}
\left\|\mathcal{F}^{-1}\left(\frac{\widehat{f}(\xi, \tau)}{\tau+|\xi|^{2}+i 2^{-k}}\right)\right\|_{L_{t, x}^{2}(|V|)}^{2} & \leq[V]^{2} \int\|\widehat{f(x, \cdot)}(\tau)\|_{L_{x}^{2}\left(|V|^{-1}\right)}^{2} d \tau \\
& \leq[V]^{2}\|f\|_{L_{t, x}^{2}\left(|V|^{-1}\right)}^{2}
\end{aligned}
$$

as desired. Next, we will obtain

$$
\left\|\mathcal{F}^{-1}\left(\frac{\left(2^{-k}-\xi_{n}\right) \widehat{f}(\xi, \tau)}{\left(\tau+|\xi|^{2}+i \xi_{n}\right)\left(\tau+|\xi|^{2}+i 2^{-k}\right)}\right)\right\|_{L_{t, x}^{2}(|V|)} \leq C[V]\|f\|_{L_{t, x}^{2}\left(|V|^{-1}\right)}
$$

under the condition that supp $\widehat{f} \subset\left\{(\xi, \tau) \in \mathbb{R}^{n+1}:\left|\xi_{n}\right| \sim 2^{-k}\right\}$. Then, by noting that

$$
\frac{1}{\tau+|\xi|^{2}+i \xi_{n}}=\frac{1}{\tau+|\xi|^{2}+i 2^{-k}}+i \frac{\left(2^{-k}-\xi_{n}\right) \widehat{f}(\xi, \tau)}{\left(\tau+|\xi|^{2}+i \xi_{n}\right)\left(\tau+|\xi|^{2}+i 2^{-k}\right)},
$$

the above two estimates (5.12) and (5.13) would imply the desired estimate (5.9).

For (5.13) we make use of the weighted $L^{2}$ estimate for the Schrödinger propagator in Proposition 4.1. By changing variables $\tau+|\xi|^{2} \rightarrow \rho$ in (5.13), we need to show that

$$
\left\|\int_{\mathbb{R}} e^{i t \rho} \int_{\mathbb{R}} e^{i(t-s) \Delta} F(\cdot, s) d s d \rho\right\|_{L_{t, x}^{2}(|V|)} \leq C[V]\|f\|_{L_{t, x}^{2}\left(|V|^{-1}\right)},
$$

where

$$
\widehat{F(\cdot, s)}(\xi)=\frac{\left(2^{-k}-\xi_{n}\right)}{\left(\rho+i \xi_{n}\right)\left(\rho+i 2^{-k}\right)} e^{-i s \rho} \widehat{f(\cdot, s)}(\xi) .
$$

Using Minkowski's inequality and Proposition 4.1, the left-hand side of (5.14) is bounded by

$$
C[V] \int_{\mathbb{R}}\left\|\mathcal{F}^{-1}\left(\frac{2^{-k}-\xi_{n}}{\left(\rho+i \xi_{n}\right)\left(\rho+i 2^{-k}\right)} e^{-i s \rho} \widehat{f(\cdot, s)}(\xi)\right)\right\|_{L_{t, x}^{2}\left(|V|^{-1}\right)} d \rho .
$$


Now, let us set

$$
m_{k, \rho}\left(\xi_{n}\right)=\frac{2^{-k}-\xi_{n}}{\left(\rho+i \xi_{n}\right)\left(\rho+i 2^{-k}\right)} .
$$

Then it is an elementary matter to check

$$
\left|m_{k, \rho}\left(\xi_{n}\right)\right| \leq \frac{C 2^{-k}}{\rho^{2}+2^{-2 k}}
$$

and

$$
\left|\frac{\partial m_{k, \rho}\left(\xi_{n}\right)}{\partial \xi_{n}}\right| \leq \frac{C}{\rho^{2}+2^{-2 k}}
$$

since we are assuming that $\operatorname{supp} \widehat{f} \subset\left\{(\xi, \tau) \in \mathbb{R}^{n+1}:\left|\xi_{n}\right| \sim 2^{-k}\right\}$. So, $m_{k, \rho}\left(\xi_{n}\right)$ satisfies the conditions of the Marcinkiewicz multiplier theorem. Since $|V|^{-1} \in$ $A_{2}(\mathbb{R})$ in the $x_{n}$ variable uniformly in other variables, by the multiplier theorem on weighted $L^{2}$ spaces with weights in the $A_{2}$ class (see Theorem 2 in [25]), (5.15) is bounded by

$$
C[V] \int_{\mathbb{R}} \frac{2^{-k}}{\rho^{2}+2^{-2 k}}\|f\|_{L_{t, x}^{2}\left(|V|^{-1}\right)} d \rho .
$$

Consequently we get (5.14) since $2^{-k} /\left(\rho^{2}+2^{-2 k}\right) \in L^{1}$ uniformly in $k \in \mathbb{Z}$. This completes the proof.

Comment on the $A_{2}$ assumption. Given a class $\mathcal{R}$ of resolvent type, the Sobolev type inequality (5.4) clearly holds for $V \in \mathcal{R}$ with $[V]$ replaced by $[V]_{\mathcal{R}}$. We point out that the $A_{2}$ assumption in (5.4) is superfluous in the cases of $\mathcal{R}=L^{n / 2}, L^{n / 2, \infty}$, or $\mathcal{L}^{2, p}, p>(n-1) / 2$, and so the Carleman estimate does not also need this assumption in these cases. Since $[V]_{\mathcal{R}}$ is given by the usual norm for these cases, we only need to consider the case of $\mathcal{R}=\mathcal{L}^{2, p}, p>(n-1) / 2$, because $\|V\|_{L^{n / 2}} \sim$ $\|V\|_{\mathcal{L}^{2, n / 2}}$ and $\|V\|_{\mathcal{L}^{2, p}} \leq C\|V\|_{L^{n / 2, \infty}}$. (Recall that $L^{n / 2}=\mathcal{L}^{2, p}$ for $p=n / 2$ and $L^{n / 2, \infty} \subset \mathcal{L}^{2, p}$ if $p<n / 2$.) In this case, due to a good property of $\mathcal{L}^{2, p}$, more tools are available for removing the $A_{2}$ assumption ( $c f$. [8, 37]).

First, recall from (1.5) the definition of $A_{p}$ weights for $1<p<\infty$. Also, $w$ is said to be of class $A_{1}$ if there is a constant $C_{A_{1}}$ such that for almost every $x$

$$
M(w)(x) \leq C_{A_{1}} w(x),
$$

where $M(w)$ is the Hardy-Littlewood maximal function of $w$ given by

$$
M(w)(x)=\sup _{Q \ni x} \frac{1}{|Q|} \int_{Q} w(y) d y
$$

with cubes $Q$ in $\mathbb{R}^{n}$. Then, the following is one of the basic properties of $A_{p}$ weights (cf. [16]):

$$
A_{p} \subset A_{q}, \quad 1 \leq p<q<\infty,
$$

and $C_{A_{q}}$ may be taken to be less than $C_{A_{p}}$. It is also known that

$$
(M(w))^{\delta} \in A_{1}, \quad 0<\delta<1,
$$

with $C_{A_{1}}$ independent of $w$ if $M(w)(x)<\infty$ for almost every $x$. (See 39], V, Subsection 5.2, and also Proposition 2 in [9].) Next, consider the following onedimensional maximal function of $V$ in the $x_{n}$ variable:

$$
\tilde{V}(x)=\left(\sup _{\mu} \frac{1}{2 \mu} \int_{x_{n}-\mu}^{x_{n}+\mu}\left|V\left(x_{1}, \ldots, x_{n-1}, \lambda\right)\right|^{\beta} d \lambda\right)^{1 / \beta}, \quad \beta>1 .
$$


Namely, $\tilde{V}(x)=M\left(\left|V\left(x^{\prime}, \cdot\right)\right|^{\beta}\right)^{1 / \beta}\left(x_{n}\right)$, where $x^{\prime}=\left(x_{1}, \ldots, x_{n-1}\right)$. If $\beta \leq p$, by Hölder's inequality in $\lambda$ and the fact that $V \in \mathcal{L}^{2, p}$, it follows that

$$
\widetilde{V}(x) \leq C\left(\sup _{\mu} \frac{1}{2 \mu} \int_{x_{n}-\mu}^{x_{n}+\mu}\left|V\left(x^{\prime}, \lambda\right)\right|^{p} d \lambda\right)^{1 / p}<\infty,
$$

and so $M\left(\left|V\left(x^{\prime}, \cdot\right)\right|^{\beta}\right)\left(x_{n}\right)<\infty$ for almost every $x_{n}$. Thus it follows from (5.17) that

$$
\widetilde{V}=M\left(\left|V\left(x^{\prime}, \cdot\right)\right|^{\beta}\right)^{1 / \beta} \in A_{1}(\mathbb{R}),
$$

which in turn implies that $\widetilde{V} \in A_{2}(\mathbb{R})$ (see (5.16) $)$ in the $x_{n}$ variable uniformly in $x^{\prime}$. Finally, if $V \in \mathcal{L}^{2, p}$ for $p>(n-1) / 2$, and $\beta<p$, then

$$
\widetilde{V} \in \mathcal{L}^{2, p} \quad \text { and } \quad\|\widetilde{V}\|_{\mathcal{L}^{2, p}} \leq C\|V\|_{\mathcal{L}^{2, p}}
$$

which can be found in 8 . (See Lemma 2.14 there.)

Consequently, if $V \in \mathcal{L}^{2, p}$ for $p>(n-1) / 2$, then so is $\widetilde{V}$. Also, since $\widetilde{V} \in A_{2}(\mathbb{R})$ in the $x_{n}$ variable uniformly in $x^{\prime}$, it now follows that

$$
\|u\|_{L_{t, x}^{2}(\widetilde{V})} \leq C\|\widetilde{V}\|_{\mathcal{L}^{2, p}}\left\|\left(i \partial_{t}+\Delta+P(D)\right) u\right\|_{L_{t, x}^{2}\left(\widetilde{V}^{-1}\right)} .
$$

Since $|V| \leq \widetilde{V}$ and $\|\widetilde{V}\|_{\mathcal{L}^{2, p}} \leq C\|V\|_{\mathcal{L}^{2, p}}$ (see (15.18) $)$, this readily implies the desired inequality

$$
\|u\|_{L_{t, x}^{2}(|V|)} \leq C\|V\|_{\mathcal{L}^{2, p}}\left\|\left(i \partial_{t}+\Delta+P(D)\right) u\right\|_{L_{t, x}^{2}\left(|V|^{-1}\right)}
$$

for the original $V$ without the $A_{2}$ assumption.

\section{Global unique continuation}

Given a class $\mathcal{R}$ of resolvent type, from Theorem 1.4, one can immediately see that the unique continuation for the Schrödinger inequality

$$
\left|\left(i \partial_{t}+\Delta\right) u(x, t)\right| \leq|V(x) u(x, t)|
$$

holds globally from a half-space in $\mathbb{R}^{n+1}$ under suitable assumptions on the potential $V \in \mathcal{R}$. This shows a direct link between the unique continuation and the resolvent estimate, and so a few implications of the resolvent estimates in Section 2 are straightforward. For instance, one can obtain some new results on the unique continuation with $V \in \mathcal{S}_{n}$. Recall that $\mathcal{L}^{2, p} \subset \mathcal{S}_{n}$ for $p>(n-1) / 2$, and so $L^{n / 2}, L^{n / 2, \infty} \subset \mathcal{S}_{n}$. In particular, $\mathcal{K}, \mathcal{R} \subset \mathcal{S}_{3}$. (See the paragraph below Definition 2.1.)

Making use of the Carleman estimate in Proposition 5.1, we will prove in this section the following global theorem, Theorem 1.4.

Theorem 6.1 (Theorem 1.4). Let $n \geq 2$. Assume that $u \in \mathcal{H}_{t}^{1}\left(\mathcal{L}_{2}\right) \cap \mathcal{H}_{x}^{2}\left(\mathcal{L}_{2}\right)$ is a solution of (6.1) with $V \in \mathcal{R}$ and vanishes in a half-space with a unit normal vector $\nu \in \mathbb{R}^{n+1}$. Then it must be identically zero if $|V| \in A_{2}\left(\nu^{\prime}\right)$ and $[V]_{\mathcal{R}}<\varepsilon$ for a sufficiently small $\varepsilon>0$.

First, note from the previous section that the $A_{2}$ assumption in Proposition 5.1 is superfluous in the cases of $\mathcal{R}=L^{n / 2}, L^{n / 2, \infty}$, or $\mathcal{L}^{2, p}, p>(n-1) / 2$. Hence, as mentioned earlier, the smallness assumption can be given in these cases by a more local one,

$$
\sup _{a \in \mathbb{R}} \lim _{\delta \rightarrow 0}\left[\chi_{S_{a, a+\delta}\left(\nu^{\prime}\right)} V\right]_{\mathcal{R}}<\varepsilon \quad\left(\text { if } \nu=\left(\nu^{\prime}, 0\right)\right)
$$


which is trivially satisfied for the case $\mathcal{R}=L^{n / 2}$, and the solution space $\mathcal{L}_{2}$ can be extended to the whole space $L^{2}$ in the cases of $\mathcal{R}=L^{n / 2, \infty}, \mathcal{L}^{2, p},(n-1) / 2<$ $p<n / 2$, or $\mathcal{S}_{3}$. In this regard, the Fefferman-Phong class $\mathcal{L}^{2, p}$ is especially good for our theorems, and it is worth summarizing that point in the following theorem separately. What we have just remarked above will be clearly demonstrated through its proof.

Theorem 6.2. Let $V \in \mathcal{L}^{2, p}$ for $(n-1) / 2<p<n / 2, n \geq 3$. Assume that $u \in \mathcal{H}_{t}^{1}\left(L^{2}\right) \cap \mathcal{H}_{x}^{2}\left(L^{2}\right)$ is a solution of (6.1) and vanishes in a half-space with a unit normal vector $\nu \in \mathbb{R}^{n+1}$. Then it must be identically zero if $\|V\|_{\mathcal{L}^{2, p}}<\varepsilon$ for a sufficiently small $\varepsilon>0$. If $\nu=\left(\nu^{\prime}, 0\right) \in \mathbb{R}^{n+1}$, then the smallness assumption can be replaced by

$$
\sup _{a \in \mathbb{R}} \lim _{\delta \rightarrow 0}\left\|\chi_{S_{a, a+\delta}\left(\nu^{\prime}\right)} V\right\|_{\mathcal{L}^{2, p}}<\varepsilon
$$

which is a weaker one.

The rest of this section is devoted to proving the above-mentioned theorems.

Proof of Theorem 6.1. By translation we may first assume that the solution $u$ vanishes in the half-space $\left\{(x, t) \in \mathbb{R}^{n+1}:\langle(x, t), \nu\rangle>0\right\}$. Now, from induction it suffices to show that there is $\sigma>0$ so that $u=0$ in the strip

$$
S_{-\sigma, 0}(\nu)=\left\{(x, t) \in \mathbb{R}^{n+1}:-\sigma<\langle(x, t), \nu\rangle \leq 0\right\}
$$

with width $\sigma>0$. We will show this by making use of the Carleman estimate in Proposition 5.1 .

First, let $\psi: \mathbb{R}^{n+1} \rightarrow[0, \infty)$ be a smooth function such that $\operatorname{supp} \psi \subset B(0,1)$ and

For $0<\varepsilon<1$, we put

$$
\int_{\mathbb{R}^{n+1}} \psi(x, t) d x d t=1 .
$$

$$
\psi_{\varepsilon}(x, t)=\varepsilon^{-(n+1)} \psi(x / \varepsilon, t / \varepsilon) .
$$

Also, let $\phi: \mathbb{R}^{n+1} \rightarrow[0,1]$ be a smooth function such that $\phi=1$ in $B(0,1)$ and $\phi=0$ in $\mathbb{R}^{n+1} \backslash B(0,2)$, and for $R \geq 1$ we set $\phi_{R}(x, t)=\phi(x / R, t / R)$.

Now we apply the Carleman estimate (5.2) for $V \in \mathcal{R}$ to the $C_{0}^{\infty}$ function

$$
\widetilde{u}(x, t)=\left(u * \psi_{\varepsilon}\right)(x, t) \phi_{R}(x, t)
$$

which is supported in the set

$$
H_{\varepsilon}=\left\{(x, t) \in \mathbb{R}^{n+1}:\langle(x, t), \nu\rangle \leq \varepsilon\right\} .
$$

Then, we see that

$$
\left\|\chi_{S_{-\sigma, 0}(\nu)} e^{\beta\langle(x, t), \nu\rangle} \widetilde{u}\right\|_{L_{t, x}^{2}(|V|)} \leq C[V]_{\mathcal{R}}\left\|\chi_{H_{\varepsilon}} e^{\beta\langle(x, t), \nu\rangle}\left(i \partial_{t}+\Delta\right) \widetilde{u}\right\|_{L_{t, x}^{2}\left(|V|^{-1}\right)} .
$$

By using Fatou's lemma it now follows that

$$
\begin{aligned}
\| \chi_{S_{-\sigma, 0}(\nu)} e^{\beta\langle(x, t), \nu\rangle} & u \phi_{R} \|_{L_{t, x}^{2}(|V|)} \\
& \leq C[V]_{\mathcal{R}} \lim _{\varepsilon \rightarrow 0}\left\|\chi_{H_{\varepsilon}} e^{\beta\langle(x, t), \nu\rangle}\left(i \partial_{t}+\Delta\right) \widetilde{u}\right\|_{L_{t, x}^{2}\left(|V|^{-1}\right)},
\end{aligned}
$$

and note that

$$
\left(i \partial_{t}+\Delta\right) \widetilde{u}=\left(\left(i \partial_{t}+\Delta\right) u * \psi_{\varepsilon}\right) \phi_{R}+\left(u * \psi_{\varepsilon}\right)\left(i \partial_{t}+\Delta\right) \phi_{R}+2 \nabla_{x}\left(u * \psi_{\varepsilon}\right) \cdot \nabla_{x} \phi_{R} .
$$


Hence, since we are assuming that $u \in \mathcal{H}_{t}^{1}\left(\mathcal{L}_{2}\right) \cap \mathcal{H}_{x}^{2}\left(\mathcal{L}_{2}\right)$, it follows that

$$
\begin{aligned}
\lim _{\varepsilon \rightarrow 0} \| \chi_{H_{\varepsilon}} e^{\beta\langle(x, t), \nu\rangle}\left(i \partial_{t}+\Delta\right) \widetilde{u} & \|_{L_{t, x}^{2}\left(|V|^{-1}\right)} \\
& \leq\left\|\chi_{H} e^{\beta\langle(x, t), \nu\rangle}\left(\left(i \partial_{t}+\Delta\right) u\right) \phi_{R}\right\|_{L_{t, x}^{2}\left(|V|^{-1}\right)} \\
& +\left\|\chi_{H} e^{\beta\left\langle(x, t), \nu_{0}\right\rangle} u\left(i \partial_{t}+\Delta\right) \phi_{R}\right\|_{L_{t, x}^{2}\left(|V|^{-1}\right)} \\
& +2\left\|\chi_{H} e^{\beta\left\langle(x, t), \nu_{0}\right\rangle} \nabla_{x} u \cdot \nabla_{x} \phi_{R}\right\|_{L_{t, x}^{2}\left(|V|^{-1}\right)}
\end{aligned}
$$

where

$$
H=\left\{(x, t) \in \mathbb{R}^{n+1}:\langle(x, t), \nu\rangle \leq 0\right\} .
$$

Then, by letting $R \rightarrow \infty$ we see that

$$
\begin{gathered}
\lim _{R \rightarrow \infty} \lim _{\varepsilon \rightarrow 0}\left\|\chi_{H_{\varepsilon}} e^{\beta\langle(x, t), \nu\rangle}\left(i \partial_{t}+\Delta\right) \widetilde{u}\right\|_{L_{t, x}^{2}\left(|V|^{-1}\right)} \\
\leq\left\|\chi_{H} e^{\beta\langle(x, t), \nu\rangle}\left(i \partial_{t}+\Delta\right) u\right\|_{L_{t, x}^{2}\left(|V|^{-1}\right)}
\end{gathered}
$$

By applying Fatou's lemma to (6.8) and using this, we conclude that

$$
\left\|\chi_{S_{-\sigma, 0}(\nu)} e^{\beta\langle(x, t), \nu\rangle} u\right\|_{L_{t, x}^{2}(|V|)} \leq C[V]_{\mathcal{R}}\left\|\chi_{H} e^{\beta\langle(x, t), \nu\rangle}\left(i \partial_{t}+\Delta\right) u\right\|_{L_{t, x}^{2}\left(|V|^{-1}\right)^{\prime}}
$$

Now we decompose the norm in the right-hand side of (6.11) into two parts,

$$
\left\|\chi_{S_{-\sigma, 0}(\nu)} e^{\beta\langle(x, t), \nu\rangle}\left(i \partial_{t}+\Delta\right) u\right\|_{L_{t, x}^{2}\left(|V|^{-1}\right)}
$$

and

$$
\left\|\chi_{H \backslash S_{-\sigma, 0}(\nu)} e^{\beta\langle(x, t), \nu\rangle}\left(i \partial_{t}+\Delta\right) u\right\|_{L_{t, x}^{2}\left(|V|^{-1}\right)} .
$$

Then, since $u$ is a solution of the Schrödinger inequality and we are assuming that $[V]_{\mathcal{R}}<\varepsilon$, the first part can be absorbed into the left-hand side of (6.11) in the following way:

$$
\begin{aligned}
\left\|\chi_{S_{-\sigma, 0}(\nu)} e^{\beta\langle(x, t), \nu\rangle}\left(i \partial_{t}+\Delta\right) u\right\|_{L_{t, x}^{2}\left(|V|^{-1}\right)} & \leq\left\|\chi_{S_{-\sigma, 0}(\nu)} e^{\beta\langle(x, t), \nu\rangle} V u\right\|_{L_{t, x}^{2}\left(|V|^{-1}\right)} \\
& =\left\|\chi_{S_{-\sigma, 0}(\nu)} e^{\beta\langle(x, t), \nu\rangle} u\right\|_{L_{t, x}^{2}(|V|)} .
\end{aligned}
$$

On the other hand, the second part is bounded for $\beta>0$ by

$$
e^{-\beta \sigma}\left\|\chi_{H \backslash S_{-\sigma, 0}(\nu)}\left(i \partial_{t}+\Delta\right) u\right\|_{L_{t, x}^{2}\left(|V|^{-1}\right)}
$$

because $\langle(x, t), \nu\rangle \leq-\sigma$ in the set $H \backslash S_{-\sigma, 0}(\nu)$. Consequently, we get

$$
\begin{aligned}
\left\|\chi_{S_{-\sigma, 0}(\nu)} e^{\beta(\langle(x, t), \nu\rangle+\sigma)} u\right\|_{L_{t, x}^{2}(|V|)} & \leq C\left\|\chi_{H \backslash S_{-\sigma, 0}(\nu)}\left(i \partial_{t}+\Delta\right) u\right\|_{L_{t, x}^{2}\left(|V|^{-1}\right)} \\
& <\infty .
\end{aligned}
$$

Finally, by letting $\beta \rightarrow \infty$ it follows that $u=0$ in the strip $S_{-\sigma, 0}(\nu)$. This completes the proof.

Proof of Theorem 6.2. To prove the first assertion of the theorem, we only need to show that the solution space $\mathcal{L}_{2}$ can be extended to the whole space $L^{2}$. For this we will use the fact that $1 /|x|^{2} \in \mathcal{L}^{2, p}$ and the Fefferman-Phong inequality (2.4). First, note that

$$
\left\|(f * g) \chi_{B(0,2 R)}\right\|_{L_{t, x}^{2}\left(|V|^{-1}\right)} \leq C R^{2}\|f\|_{L_{t, x}^{2}}\|g\|_{L_{t, x}^{1}}
$$


if $|V| \geq C /|x|^{2}$ for $|x| \leq 2 R$. Since we can choose $\delta>0$ small enough so that $\left\|\delta /|x|^{2}\right\|_{\mathcal{L}^{2, p}}=\delta\left\|1 /|x|^{2}\right\|_{\mathcal{L}^{2, p}}$ is sufficiently small and

$$
\left|\left(i \partial_{t}+\Delta\right) u\right| \leq|V u| \leq\left|\left(|V|+\delta /|x|^{2}\right) u\right|,
$$

we may assume that $|V| \geq \delta /|x|^{2}$ by replacing $V$ with $|V|+\delta /|x|^{2}$. Then, by using (6.13), one can get (6.9) assuming $u \in \mathcal{H}_{t}^{1}\left(L^{2}\right) \cap \mathcal{H}_{x}^{2}\left(L^{2}\right)$ instead of $u \in$ $\mathcal{H}_{t}^{1}\left(\mathcal{L}_{2}\right) \cap \mathcal{H}_{x}^{2}\left(\mathcal{L}_{2}\right)$. Next, one can use the Fefferman-Phong inequality (2.4) to bound the right-hand side of (6.12). Indeed, by applying the inequality, it follows that

$$
\begin{aligned}
\left\|\chi_{H \backslash S_{-\sigma, 0}(\nu)}\left(i \partial_{t}+\Delta\right) u\right\|_{L_{t, x}^{2}\left(|V|^{-1}\right)} & \leq\|V u\|_{L_{t, x}^{2}\left(|V|^{-1}\right)} \\
& =\|u\|_{L_{t, x}^{2}(|V|)} \\
& \leq C\|V\|_{\mathcal{L}^{2, p}}\|\nabla u\|_{L_{t, x}^{2}}<\infty .
\end{aligned}
$$

Thus, the previous proof entirely works for $u \in \mathcal{H}_{t}^{1}\left(L^{2}\right) \cap \mathcal{H}_{x}^{2}\left(L^{2}\right)$.

For the second assertion of the theorem, we consider the following $C_{0}^{\infty}$ function instead of (6.6):

$$
\widetilde{u}_{\sigma}(x, t)=\left(u * \psi_{\varepsilon}\right)(x, t) \phi_{R}(x, t) \varphi_{\sigma}(\langle(x, t), \nu\rangle),
$$

where $\varphi_{\sigma}(r)=\varphi(r / \sigma)$ for $\varphi: \mathbb{R} \rightarrow[0,1]$ which is a smooth function equal to 1 in $\{-1 / 2<r \leq 0\}$ and equal to 0 in $\{r<-1$ or $r \geq 1 / 2\}$. Note first that if $\nu=\left(\nu^{\prime}, 0\right)$, then

$$
S_{a, a+\delta}(\nu)=\mathbb{R} \times S_{a, a+\delta}\left(\nu^{\prime}\right),
$$

and so

$$
\left\|\chi_{S_{a, a+\delta}(\nu)}(x, t) e^{\beta\langle(x, t), \nu\rangle} \widetilde{u}_{\sigma}\right\|_{L_{t, x}^{2}(|V|)}=\left\|e^{\beta\langle(x, t), \nu\rangle} \widetilde{u}_{\sigma}\right\|_{L_{t, x}^{2}\left(\left|\chi_{S_{a, a+\delta}\left(\nu^{\prime}\right)}(x) V\right|\right)} .
$$

Then, since $\widetilde{u}_{\sigma}$ is supported in the strip $S_{-\sigma, \varepsilon}(\nu)$, by applying the Carleman estimate (5.2) with $V=\chi_{S_{-3 \sigma / 2, \sigma / 2}\left(\nu^{\prime}\right)} V$ to $\widetilde{u}_{\sigma}$, one can see that

$$
\begin{aligned}
& \left\|\chi_{S_{-\sigma / 2,0}(\nu)} e^{\beta\langle(x, t), \nu\rangle} \widetilde{u}_{\sigma}\right\|_{L_{t, x}^{2}(|V|)} \\
& \quad \leq C\left\|\chi_{S_{-3 \sigma / 2, \sigma / 2}\left(\nu^{\prime}\right)} V\right\|_{\mathcal{L}^{2, p}}\left\|\chi_{S_{-\sigma, \varepsilon}(\nu)} e^{\beta\langle(x, t), \nu\rangle}\left(i \partial_{t}+\Delta\right) \widetilde{u}_{\sigma}\right\|_{L_{t, x}^{2}\left(|V|^{-1}\right)}
\end{aligned}
$$

because $S_{-\sigma, \varepsilon}\left(\nu^{\prime}\right) \subset S_{-3 \sigma / 2, \sigma / 2}\left(\nu^{\prime}\right)$ for $\varepsilon<\sigma / 2$. Hence, by the same limiting argument as before, it follows that

$$
\begin{aligned}
& \left\|\chi_{S_{-\sigma / 2,0}(\nu)} e^{\beta\langle(x, t), \nu\rangle} u\right\|_{L_{t, x}^{2}(|V|)} \\
& \quad \leq C\left\|\chi_{S_{-3 \sigma / 2, \sigma / 2}\left(\nu^{\prime}\right)} V\right\|_{\mathcal{L}^{2, p}}\left\|\chi_{S_{-\sigma, 0}(\nu)} e^{\beta\langle(x, t), \nu\rangle}\left(i \partial_{t}+\Delta\right) u\right\|_{L_{t, x}^{2}\left(|V|^{-1}\right)}
\end{aligned}
$$

Now, from the smallness assumption (6.3) we can choose $\sigma>0$ small enough so that

$$
C\left\|\chi_{S_{-3 \sigma / 2, \sigma / 2}\left(\nu^{\prime}\right)} V\right\|_{\mathcal{L}^{2, p}}<1 / 2 \text {. }
$$

By decomposing $\chi_{S_{-\sigma, 0}(\nu)}=\chi_{S_{-\sigma / 2,0}(\nu)}+\chi_{S_{-\sigma,-\sigma / 2}(\nu)}$ and repeating the previous argument, this leads to

$$
\begin{aligned}
\left\|\chi_{S_{-\sigma / 2,0}(\nu)} e^{\beta(\langle(x, t), \nu\rangle+\sigma / 2)} u\right\|_{L_{t, x}^{2}(|V|)} & \leq C\left\|\chi_{S_{-\sigma,-\sigma / 2}(\nu)}\left(i \partial_{t}+\Delta\right) u\right\|_{L_{t, x}^{2}\left(|V|^{-1}\right)} \\
& <\infty .
\end{aligned}
$$

Hence, by letting $\beta \rightarrow \infty$ it follows that $u=0$ in the strip $S_{-\sigma / 2,0}(\nu)$. This completes the proof. 


\section{LOCAL UNiQUE CONTINUATION}

Now we turn to the local unique continuation theorem. Recall that there exists a smooth potential $V$ such that $\left(i \partial_{t}+\Delta\right) u=V(x, t) u, 0 \in \operatorname{supp} u$, and $u=0$ on $\left\{(x, t) \in \mathbb{R}^{n+1}: x_{1}<0\right\}$ in a neighborhood of the origin. See the paragraph above Theorem 1.5. Since $0 \in \operatorname{supp} u$, the solution $u$ cannot vanish near the origin across the hyperplane $\left\{(x, t) \in \mathbb{R}^{n+1}: x_{1}=0\right\}$. This shows that the Schrödinger equation does not have, as a rule, a property of unique continuation locally across a hyperplane in $\mathbb{R}^{n+1}$. However, our result in Theorem 1.5 says that the unique continuation for the Schrödinger inequality

$$
\left|\left(i \partial_{t}+\Delta\right) u(x, t)\right| \leq|V(x) u(x, t)|
$$

can hold locally across a hypersurface on a sphere in $\mathbb{R}^{n+1}$ into an interior region of the sphere.

Theorem 7.1 (Theorem[1.5). Let $n \geq 2$ and let $S_{r}^{n}$ be a sphere in $\mathbb{R}^{n+1}$ with radius $r$. Assume that $u \in \mathcal{H}_{t}^{1}\left(\mathcal{L}_{2}\right) \cap \mathcal{H}_{x}^{2}\left(\mathcal{L}_{2}\right)$ is a solution of (7.1) with $V \in \mathcal{R}$ and that it vanishes on an exterior neighborhood of $S_{r}^{n}$ in a neighborhood of a point $p \in S_{r}^{n}$. Let $\nu$ be the unit outward normal vector of $S_{r}^{n}$ at $p$. Then it follows that $u \equiv 0$ in a neighborhood of $p$, if $|V| \in A_{2}\left(\nu^{\prime}\right)$ and $[V]_{\mathcal{R}}<\varepsilon$ for a sufficiently small $\varepsilon>0$.

In the same cases as in the global theorem, the solution space $\mathcal{L}_{2}$ can be extended to the whole space $L^{2}$ and the smallness assumption can be given by a more local one,

$$
\lim _{\delta \rightarrow 0}\left[\chi_{B_{\delta}\left(p^{\prime}\right)} V\right]_{\mathcal{R}}<\varepsilon
$$

where $B_{\delta}(p)$ denotes a ball centered at $p$ with radius $\delta$. Unlike (6.2), we do not need to assume $\nu=\left(\nu^{\prime}, 0\right)$, since the property (6.14) is trivially satisfied for balls in the sense that $x \in B_{\delta}\left(p^{\prime}\right)$ whenever $(x, t) \in B_{\delta}(p)$.

Proof of Theorem 7.1. For simplicity of notation, we shall first assume that the point $p$ is the origin. Then, we are assuming that the solution $u$ vanishes on an exterior neighborhood of a sphere $S_{r}^{n}$ in a neighborhood $\mathcal{N}$ of the origin, and $\nu$ is the unit outward normal vector of $S_{r}^{n}$ at the origin. Hence, there is $\delta>0$ so that $B_{\delta}(0) \subset \mathcal{N}$, and since $u$ vanishes on an exterior neighborhood of $S_{r}^{n}$ in $\mathcal{N}$, we can choose $\sigma>0$ small enough so that $u$ vanishes on the set

$$
\left(B_{\delta}(0) \backslash B_{\delta / 2}(0)\right) \cap S_{-\sigma, 0}(\nu),
$$

where $S_{-\sigma, 0}(\nu)$ is the strip given in (6.4). Of course, the solution is also vanishing in the set

$$
B_{\delta}(0) \cap\left\{(x, t) \in \mathbb{R}^{n+1}:\langle(x, t), \nu\rangle>0\right\} .
$$

Now it suffices to show that $u$ vanishes in the set $B_{\delta / 2}(0) \cap S_{-\sigma, 0}(\nu)$.

To show this, we apply the Carleman estimate in Proposition 5.1 to the $C_{0}^{\infty}$ function

$$
\widetilde{u}(x, t)=\left(u * \psi_{\varepsilon}\right)(x, t) \eta(x, t),
$$

where $\psi_{\varepsilon}$ is given in (6.5) and $\eta: \mathbb{R}^{n+1} \rightarrow[0,1]$ is a smooth function such that $\eta=1$ in $B_{\delta / 2}(0)$ and $\eta=0$ in $\mathbb{R}^{n+1} \backslash B_{\delta}(0)$. In fact, since $\widetilde{u}$ is supported in the set $B_{\delta}(0) \cap H_{\varepsilon}$, where $H_{\varepsilon}$ is given in (6.7), one can see that

$$
\begin{aligned}
\| \chi_{B_{\delta / 2}(0) \cap S_{-\sigma, 0}(\nu)} e^{\beta\langle(x, t), \nu\rangle} & \widetilde{u} \|_{L_{t, x}^{2}(|V|)} \\
& \leq C[V]_{\mathcal{R}}\left\|\chi_{B_{\delta}(0) \cap H_{\varepsilon}} e^{\beta\langle(x, t), \nu\rangle}\left(i \partial_{t}+\Delta\right) \widetilde{u}\right\|_{L_{t, x}^{2}\left(|V|^{-1}\right)}
\end{aligned}
$$


Then, by the same limiting argument as before, it follows that

$$
\begin{aligned}
\left\|\chi_{B_{\delta / 2}(0) \cap S_{-\sigma, 0}(\nu)} e^{\beta\langle(x, t), \nu\rangle} u\right\|_{L_{t, x}^{2}(|V|)} & \\
& \leq C[V]_{\mathcal{R}}\left\|\chi_{B_{\delta}(0) \cap H} e^{\beta\langle(x, t), \nu\rangle}\left(i \partial_{t}+\Delta\right)(u \eta)\right\|_{L_{t, x}^{2}\left(|V|^{-1}\right)},
\end{aligned}
$$

where $H$ is given in (6.10).

Now, we decompose the norm in the right-hand side of (7.2) into two parts. Then the first part

$$
\left\|\chi_{B_{\delta / 2}(0) \cap S_{-\sigma, 0}(\nu)} e^{\beta\langle(x, t), \nu\rangle}\left(i \partial_{t}+\Delta\right) u\right\|_{L_{t, x}^{2}\left(|V|^{-1}\right)}
$$

can be absorbed into the left-hand side of (7.2) as before, while the second part

$$
\left\|\chi_{\left(B_{\delta}(0) \cap H\right) \backslash\left(B_{\delta / 2}(0) \cap S_{-\sigma, 0}(\nu)\right)} e^{\beta\langle(x, t), \nu\rangle}\left(i \partial_{t}+\Delta\right)(u \eta)\right\|_{L_{t, x}^{2}\left(|V|^{-1}\right)}
$$

is bounded for $\beta>0$ by

$$
e^{-\beta \sigma}\left\|\chi_{\left(B_{\delta}(0) \cap H\right) \backslash\left(B_{\delta / 2}(0) \cap S_{-\sigma, 0}(\nu)\right)}\left(i \partial_{t}+\Delta\right)(u \eta)\right\|_{L_{t, x}^{2}\left(|V|^{-1}\right)},
$$

since $u$ vanishes on the set $\left(B_{\delta}(0) \backslash B_{\delta / 2}(0)\right) \cap S_{-\sigma, 0}(\nu)$ and $\langle(x, t), \nu\rangle \leq-\sigma$ in the set $H \backslash S_{-\sigma, 0}(\nu)$. At this point, it is worth noting that the assumption that $u$ vanishes on an exterior neighborhood of a sphere is crucial in this second part in order to guarantee that $u$ vanishes on the set $\left(B_{\delta}(0) \backslash B_{\delta / 2}(0)\right) \cap S_{-\sigma, 0}(\nu)$. Consequently, we see that

$$
\begin{aligned}
\left\|\chi_{B_{\delta / 2}(0) \cap S_{-\sigma, 0}(\nu)} e^{\beta(\langle(x, t), \nu\rangle+\sigma)} u\right\|_{L_{t, x}^{2}(|V|)} & \leq C\left\|\left(i \partial_{t}+\Delta\right)(u \eta)\right\|_{L_{t, x}^{2}\left(|V|^{-1}\right)} \\
& <\infty
\end{aligned}
$$

since $\eta \in C_{0}^{\infty}$ and $u \in \mathcal{H}_{t}^{1}\left(\mathcal{L}_{2}\right) \cap \mathcal{H}_{x}^{2}\left(\mathcal{L}_{2}\right)$. Finally, by letting $\beta \rightarrow \infty$ it follows that $u=0$ in the set $B_{\delta / 2}(0) \cap S_{-\sigma, 0}(\nu)$, as desired. This completes the proof.

\section{Further Applications}

In this section we present a few applications of our resolvent estimates to global well-posedness of the Schrödinger equation in weighted $L^{2}$ spaces.

Let us first consider the following Cauchy problem with an initial datum $f$ and a forcing term $F$ :

$$
\left\{\begin{array}{l}
i \partial_{t} u+\Delta u=F(x, t) \\
u(x, 0)=f(x)
\end{array}\right.
$$

By Duhamel's principle, the solution is then given by

$$
u(x, t)=e^{i t \Delta} f(x)-i \int_{0}^{t} e^{i(t-s) \Delta} F(\cdot, s) d s,
$$

where the first and second terms correspond to the solutions of the homogeneous $(F=0)$ and inhomogeneous $(f=0)$ problems, respectively. Now we have the following Strichartz estimates for the solutions in weighted $L^{2}$ spaces:

Proposition 8.1. Let $n \geq 2$ and let $V: \mathbb{R}^{n} \rightarrow \mathbb{C}$ be such that the resolvent estimate

$$
\left\|R_{0}(z) f\right\|_{L^{2}(|V|)} \leq C(V)\|f\|_{L^{2}\left(|V|^{-1}\right)}
$$

holds uniformly in $z \in \mathbb{C} \backslash \mathbb{R}$. Then the following estimates hold:

$$
\left\|e^{i t \Delta} f\right\|_{L_{t, x}^{2}(|V|)} \leq C C(V)^{1 / 2}\|f\|_{2}
$$




$$
\begin{gathered}
\left\|\int_{-\infty}^{\infty} e^{-i s \Delta} F(\cdot, s) d s\right\|_{2} \leq C C(V)^{1 / 2}\|F\|_{L_{t, x}^{2}\left(|V|^{-1}\right)}, \\
\left\|\int_{0}^{t} e^{i(t-s) \Delta} F(\cdot, s) d s\right\|_{L_{t, x}^{2}(|V|)} \leq C C(V)\|F\|_{L_{t, x}^{2}\left(|V|^{-1}\right)} .
\end{gathered}
$$

Remark 8.2. Given a class $\mathcal{R}$ of resolvent type, this proposition clearly holds for $V \in \mathcal{R}$ with $C(V) \sim[V]_{\mathcal{R}}$. In the cases of $\mathcal{R}=\mathcal{L}^{2, p}, p>(n-1) / 2$, and $\mathcal{S}_{3}$, the above Strichartz estimates can be found in [34] and [3], respectively. From the resolvent estimates in Theorem 2.2, these previous results are extended to the class $\mathcal{S}_{n}$.

Proof of Proposition 8.1. We have already proved the homogeneous estimate (8.1) in Section 4 in two ways, and by duality it is equivalent to (8.2). For the inhomogeneous estimate (8.3), following the simple argument used for Proposition 2.5 in [33], we may write

$$
\int_{0}^{t} e^{i(t-s) \Delta} F(\cdot, s) d s=v(x, t)-e^{i t \Delta}\left(\int_{-\infty}^{\infty} e^{-i s \Delta}[\operatorname{sgn}(s) F(\cdot, s)] d s\right)(x),
$$

where

$$
v(x, t)=\lim _{\varepsilon \rightarrow 0} \mathcal{F}^{-1}\left(\frac{\widehat{F}(\xi, \tau)}{-|\xi|^{2}-\tau+i \varepsilon}\right)(x, t) .
$$

Combining (8.1) and (8.2), we get the desired estimate for the second term on the right-hand side of (8.4). To bound the first term, all we have to do is just to use the resolvent estimate in the same way as in (5.12). Now (8.3) is proved. But here, we point out that it can also be obtained more directly by appealing to Kato $H$-smoothing theory as in the homogeneous case. In fact the following 5 is due to Kato [18: If (4.7) in Lemma 4.4 holds, then

$$
\int_{\mathbb{R}}\left\|\int_{0}^{t} T e^{i(t-s) H} T^{*} F(\cdot, s) d s\right\|_{\mathcal{H}}^{2} d t \leq \widetilde{C}^{2} \int_{\mathbb{R}}\|F(\cdot, t)\|_{\mathcal{H}}^{2} d t .
$$

By applying this, with $H=-\Delta, \mathcal{H}=L^{2}$ and $T: f \mapsto|V|^{1 / 2} f$ as before, we can alternatively obtain (8.3).

Making use of Proposition 8.1 we now obtain the global well-posedness of the following Cauchy problem for the Schrödinger equation:

$$
\left\{\begin{array}{l}
i \partial_{t} u+\Delta u-V(x) u=F(x, t), \\
u(x, 0)=u_{0}(x) .
\end{array}\right.
$$

Theorem 8.3. Let $n \geq 2$. Given a class $\mathcal{R}$ of resolvent type, we assume that $V \in \mathcal{R}$ and $[V]_{\mathcal{R}}$ is sufficiently small. If $u_{0} \in L^{2}$ and $F \in L_{t, x}^{2}\left(|V|^{-1}\right)$, then there exists a unique solution of (8.5) in the weighted space $L_{t, x}^{2}(|V|)$. Furthermore, the solution $u$ belongs to $C_{t} L_{x}^{2}$, and satisfies

$$
\|u\|_{L^{2}(|V|)} \leq C[V]_{\mathcal{R}}^{1 / 2}\left\|u_{0}\right\|_{L^{2}}+C[V]_{\mathcal{R}}\|F\|_{L_{t, x}^{2}\left(|V|^{-1}\right)}
$$

and

$$
\sup _{t \in \mathbb{R}}\|u\|_{L_{x}^{2}} \leq C\left\|u_{0}\right\|_{L^{2}}+C[V]_{\mathcal{R}}^{1 / 2}\|F\|_{L_{t, x}^{2}\left(|V|^{-1}\right)} .
$$

\footnotetext{
${ }^{5}$ For the proof, see, for example, [28].
} 
Proof. The proof is quite standard once one has the weighted $L^{2}$ Strichartz estimates in Proposition 8.1 .

Let us first consider the following integral formulation of (8.5):

$$
u(x, t)=e^{i t \Delta} u_{0}(x)-i \int_{0}^{t} e^{i(t-s) \Delta} F(\cdot, s) d s+\Phi(u)(x, t),
$$

where

$$
\Phi(u)(x, t)=-i \int_{0}^{t} e^{i(t-s) \Delta}(V u)(\cdot, s) d s .
$$

Then, since $u_{0} \in L^{2}$ and $F \in L^{2}\left(|V|^{-1}\right)$, from Proposition 8.1 and

$$
(I-\Phi)(u)=e^{i t \Delta} u_{0}(x)-i \int_{0}^{t} e^{i(t-s) \Delta} F(\cdot, s) d s,
$$

where $I$ is the identity operator, it follows that

$$
(I-\Phi)(u) \in L^{2}(|V|) .
$$

Hence, the global existence in the theorem follows if the operator $I-\Phi$ has an inverse in the space $L^{2}(|V|)$. It is well known that this holds if the operator norm of $\Phi$ in $L^{2}(|V|)$ is strictly less than 1 . Namely, it is enough to show that

$$
\|\Phi(u)\|_{L^{2}(|V|)}<\|u\|_{L^{2}(|V|)} .
$$

But, using the inhomogeneous estimate (8.3), we can obtain this as follows:

$$
\begin{aligned}
\|\Phi(u)\|_{L^{2}(|V|)} & \leq C[V]_{\mathcal{R}}\|V u\|_{L^{2}\left(|V|^{-1}\right)} \\
& =C[V]_{\mathcal{R}}\|u\|_{L^{2}(|V|)} \\
& <\frac{1}{2}\|u\|_{L^{2}(|V|)} .
\end{aligned}
$$

Here, for the last inequality we used the smallness assumption on the quantity $[V]_{\mathcal{R}}$.

On the other hand, by applying Proposition 8.1 and (8.9) to (8.8), one can easily see that

$$
\begin{aligned}
\|u\|_{L^{2}(|V|)} & \leq C\left\|e^{i t \Delta} u_{0}\right\|_{L^{2}(|V|)}+C\left\|\int_{0}^{t} e^{i(t-s) \Delta} F(\cdot, s) d s\right\|_{L^{2}(|V|)} \\
& \leq C[V]_{\mathcal{R}}^{1 / 2}\left\|u_{0}\right\|_{L^{2}}+C[V]_{\mathcal{R}}\|F\|_{L_{t, x}^{2}\left(|V|^{-1}\right)} .
\end{aligned}
$$

Hence (8.6) is proved. To show (8.7), we will use (8.10) and the estimate (8.2). First, from (8.8), (8.2), and the simple fact that $e^{i t \Delta}$ is an isometry in $L^{2}$, one can see that

$$
\|u\|_{L_{x}^{2}} \leq C\left\|u_{0}\right\|_{L^{2}}+C[V]_{\mathcal{R}}^{1 / 2}\|F\|_{L^{2}\left(|V|^{-1}\right)}+C[V]_{\mathcal{R}}^{1 / 2}\|V u\|_{L^{2}\left(|V|^{-1}\right)} .
$$

Since $\|V u\|_{L^{2}\left(|V|^{-1}\right)}=\|u\|_{L^{2}(|V|)}$ and $[V]_{\mathcal{R}}$ is small, by combining the above inequality and (8.10) we get

$$
\|u\|_{L_{x}^{2}} \leq C\left\|u_{0}\right\|_{L^{2}}+C[V]_{\mathcal{R}}^{1 / 2}\|F\|_{L_{t, x}^{2}\left(|V|^{-1}\right)}
$$

as desired. Finally, it is an elementary matter to check that $u \in C_{t} L_{x}^{2}$. Now the proof is completed. 


\section{Concluding REMARKS}

The abstract Carleman estimate (5.2) in Proposition 5.1 can be modified to work for the case of time-dependent potentials $V(x, t)$ such that

$$
\sup _{t \in \mathbb{R}}|V(x, t)| \leq W(x) \in \mathcal{R} .
$$

Taking the sup in $t$ has sometimes been used for time-dependent potentials in other problems $(2,4,33,34])$ concerning Schrödinger equations. Since $|V(x, t)| \leq$ $W(x)$ for almost every $t$, the following corollary is an immediate consequence of Proposition 5.1 .

Corollary 9.1. Let $n \geq 2$ and let $V: \mathbb{R}^{n+1} \rightarrow \mathbb{C}$ be such that (9.1) holds. Then we have for $u \in C_{0}^{\infty}\left(\mathbb{R}^{n+1}\right)$

$$
\left\|e^{\beta\langle(x, t), \nu\rangle} u\right\|_{L_{t, x}^{2}(|V(x, t)|)} \leq C[W]\left\|e^{\beta\langle(x, t), \nu\rangle}\left(i \partial_{t}+\Delta\right) u\right\|_{L_{t, x}^{2}\left(|V(x, t)|^{-1}\right)}
$$

with a constant $C$ independent of $\beta \in \mathbb{R}$ and $\nu \in \mathbb{R}^{n+1}$, if $W \in A_{2}\left(\nu^{\prime}\right)$.

This is naturally expected to lead to unique continuation for the time-dependent potential case

$$
\left|\left(i \partial_{t}+\Delta\right) u(x, t)\right| \leq|V(x, t) u(x, t)|
$$

where $V$ satisfies (9.1). In fact the same type of argument used for time-independent potentials works clearly for this case if the assumptions on the potential in Theorems 1.4 and 1.5 are given for $W(x)$ instead of $V(x)$. As a consequence, one can obtain some new results on the unique continuation for (9.2). We omit the details.

It is also straightforward that the well-posedness result in the previous section can be applied to the case of time-dependent potentials $V(x, t)$ satisfying (9.1). This case has been studied in [34] and [3] for $\mathcal{R}=\mathcal{L}^{2, p}, p>(n-1) / 2$, and $\mathcal{S}_{3}$, respectively. These previous results are now extended to the class $\mathcal{S}_{n}$ as an immediate consequence of our resolvent estimates.

\section{REFERENCES}

[1] Shmuel Agmon, Spectral properties of Schrödinger operators and scattering theory, Ann. Scuola Norm. Sup. Pisa Cl. Sci. (4) 2 (1975), no. 2, 151-218. MR0397194 (53 \#1053)

[2] Juan A. Barceló, Jonathan M. Bennett, Anthony Carbery, Alberto Ruiz, and M. Cruz Vilela, A note on weighted estimates for the Schrödinger operator, Rev. Mat. Complut. 21 (2008), no. 2, 481-488. MR2441963 (2010a:35030)

[3] J. A. Barceló, J. M. Bennett, A. Ruiz, and M. C. Vilela, Local smoothing for Kato potentials in three dimensions, Math. Nachr. 282 (2009), no. 10, 1391-1405, DOI 10.1002/mana.200610808. MR2571701 (2010m:35078)

[4] J. A. Barcelo, A. Ruiz, and L. Vega, Weighted estimates for the Helmholtz equation and some applications, J. Funct. Anal. 150 (1997), no. 2, 356-382, DOI 10.1006/jfan.1997.3131. MR $1479544(99 a: 35033)$

[5] A. P. Calderón, Notes on singular integrals, MIT Press, Cambridge, Mass., 1961.

[6] T. Carleman, Sur un problème d'unicité pur les systèmes d'équations aux dérivées partielles à deux variables indépendantes (French), Ark. Mat., Astr. Fys. 26 (1939), no. 17, 9. MR0000334(1,55f)

[7] K. M. Case, Singular potentials, Physical Rev. (2) 80 (1950), 797-806. MR0040532(12,708a)

[8] Sagun Chanillo and Eric Sawyer, Unique continuation for $\Delta+v$ and the C. Fefferman-Phong class, Trans. Amer. Math. Soc. 318 (1990), no. 1, 275-300, DOI 10.2307/2001239. MR.958886 (90f:35050)

[9] R. R. Coifman and R. Rochberg, Another characterization of BMO, Proc. Amer. Math. Soc. 79 (1980), no. 2, 249-254, DOI 10.2307/2043245. MR565349(81b:42067) 
[10] Elena Cordero and Fabio Nicola, Sharpness of some properties of Wiener amalgam and modulation spaces, Bull. Aust. Math. Soc. 80 (2009), no. 1, 105-116, DOI 10.1017/S0004972709000070. MR2520527 (2010f:42046)

[11] Piero D'Ancona and Luca Fanelli, Strichartz and smoothing estimates of dispersive equations with magnetic potentials, Comm. Partial Differential Equations 33 (2008), no. 4-6, 1082-1112, DOI 10.1080/03605300701743749. MR2424390(2009i:35257)

[12] Donatella Danielli, A Fefferman-Phong type inequality and applications to quasilinear subelliptic equations, Potential Anal. 11 (1999), no. 4, 387-413, DOI 10.1023/A:1008674906902. MR.1719837 (2000j:35044)

[13] Charles L. Fefferman, The uncertainty principle, Bull. Amer. Math. Soc. (N.S.) 9 (1983), no. 2, 129-206, DOI 10.1090/S0273-0979-1983-15154-6. MR707957 (85f:35001)

[14] Damiano Foschi, Inhomogeneous Strichartz estimates, J. Hyperbolic Differ. Equ. 2 (2005), no. 1, 1-24, DOI 10.1142/S0219891605000361. MR2134950(2006a:35043)

[15] I. M. Gel'fand and G. E. Shilov, Generalized functions. Vol. I: Properties and operations, Translated by Eugene Saletan, Academic Press, New York-London, 1964. MR0166596 (29 \#3869)

[16] Loukas Grafakos, Modern Fourier analysis, 2nd ed., Graduate Texts in Mathematics, vol. 250, Springer, New York, 2009. MR2463316(2011d:42001)

[17] Alexandru D. Ionescu and Carlos E. Kenig, $L^{p}$ Carleman inequalities and uniqueness of solutions of nonlinear Schrödinger equations, Acta Math. 193 (2004), no. 2, 193-239, DOI 10.1007/BF02392564. MR2134866(2005m:35283)

[18] Tosio Kato, Wave operators and similarity for some non-selfadjoint operators, Math. Ann. 162 (1965/1966), 258-279. MR0190801 (32 \#8211)

[19] Tosio Kato and Kenji Yajima, Some examples of smooth operators and the associated smoothing effect, Rev. Math. Phys. 1 (1989), no. 4, 481-496, DOI 10.1142/S0129055X89000171. MR.1061120 (91i:47013)

[20] Markus Keel and Terence Tao, Endpoint Strichartz estimates, Amer. J. Math. 120 (1998), no. 5, 955-980. MR.1646048 (2000d:35018)

[21] C. E. Kenig, A. Ruiz, and C. D. Sogge, Uniform Sobolev inequalities and unique continuation for second order constant coefficient differential operators, Duke Math. J. 55 (1987), no. 2, 329-347, DOI 10.1215/S0012-7094-87-05518-9. MR.894584 (88d:35037)

[22] Carlos E. Kenig and Christopher D. Sogge, A note on unique continuation for Schrödinger's operator, Proc. Amer. Math. Soc. 103 (1988), no. 2, 543-546, DOI 10.2307/2047176. MR943081(89i:35043)

[23] Carlos E. Kenig and Peter A. Tomas, On conjectures of Rivière and Strichartz, Bull. Amer. Math. Soc. (N.S.) 1 (1979), no. 4, 694-697, DOI 10.1090/S0273-0979-1979-14674-3. MR.532556 (81g:42019)

[24] R. Kerman and E. Sawyer, The trace inequality and eigenvalue estimates for Schrödinger operators (English, with French summary), Ann. Inst. Fourier (Grenoble) 36 (1986), no. 4, 207-228. MR867921 (88b:35150)

[25] Douglas S. Kurtz, Littlewood-Paley and multiplier theorems on weighted $L^{p}$ spaces, Trans. Amer. Math. Soc. 259 (1980), no. 1, 235-254, DOI 10.2307/1998156. MR.561835 (80f:42013)

[26] R. Lascar and C. Zuily, Unicité et non unicité du problème de Cauchy pour une classe d'opérateurs différentiels à caractéristiques doubles (French), Duke Math. J. 49 (1982), no. 1, 137-162. MR650374 (83e:35007)

[27] Sanghyuk Lee and Ihyeok Seo, A note on unique continuation for the Schrödinger equation, J. Math. Anal. Appl. 389 (2012), no. 1, 461-468, DOI 10.1016/j.jmaa.2011.11.067. MR2876512

[28] Kiyoshi Mochizuki, Uniform resolvent estimates for magnetic Schrödinger operators and smoothing effects for related evolution equations, Publ. Res. Inst. Math. Sci. 46 (2010), no. 4, 741-754, DOI 10.2977/PRIMS/24. MR2791005 (2012g:81070)

[29] Michael Reed and Barry Simon, Methods of modern mathematical physics. I: Functional analysis, 2nd ed., Academic Press, Inc. [Harcourt Brace Jovanovich, Publishers], New York, 1980. MR751959 (85e:46002)

[30] Michael Reed and Barry Simon, Methods of modern mathematical physics. II. Fourier analysis, self-adjointness, Academic Press [Harcourt Brace Jovanovich, Publishers], New YorkLondon, 1975. MR0493420 (58 \#12429b) 
[31] Michael Reed and Barry Simon, Methods of modern mathematical physics. IV. Analysis of operators, Academic Press [Harcourt Brace Jovanovich, Publishers], New York-London, 1978. MR.0493421 (58 \#12429c)

[32] Igor Rodnianski and Wilhelm Schlag, Time decay for solutions of Schrödinger equations with rough and time-dependent potentials, Invent. Math. 155 (2004), no. 3, 451-513, DOI 10.1007/s00222-003-0325-4. MR:2038194 (2005h:35295)

[33] Alberto Ruiz and Luis Vega, On local regularity of Schrödinger equations, Internat. Math. Res. Notices 1 (1993), 13-27, DOI 10.1155/S1073792893000029. MR1201747 (94c:35060)

[34] Alberto Ruiz and Luis Vega, Local regularity of solutions to wave equations with timedependent potentials, Duke Math. J. 76 (1994), no. 3, 913-940, DOI 10.1215/S0012-709494-07636-9. MR 1309336 (95m:35110)

[35] Ihyeok Seo, Unique continuation for the Schrödinger equation with potentials in Wiener amalgam spaces, Indiana Univ. Math. J. 60 (2011), no. 4, 1203-1227, DOI 10.1512/iumj.2011.60.4824. MR2975341

[36] Ihyeok Seo, Carleman estimates for the Schrödinger operator and applications to unique continuation, Commun. Pure Appl. Anal. 11 (2012), no. 3, 1013-1036, DOI 10.3934/cpaa.2012.11.1013. MR2968606

[37] Ihyeok Seo, Global unique continuation from a half space for the Schrödinger equation, J. Funct. Anal. 266 (2014), no. 1, 85-98, DOI 10.1016/j.jfa.2013.09.025. MR 3121722

[38] Barry Simon, Schrödinger semigroups, Bull. Amer. Math. Soc. (N.S.) 7 (1982), no. 3, 447526, DOI 10.1090/S0273-0979-1982-15041-8. MR670130(86b:81001a)

[39] Elias M. Stein, Harmonic analysis: real-variable methods, orthogonality, and oscillatory integrals. With the assistance of Timothy S. Murphy, Princeton Mathematical Series, vol. 43, Monographs in Harmonic Analysis, III. Princeton University Press, Princeton, NJ, 1993. MR.1232192 (95c:42002)

[40] Elias M. Stein and Guido Weiss, Introduction to Fourier analysis on Euclidean spaces, Princeton Mathematical Series, vol. 32, Princeton University Press, Princeton, NJ, 1971. MR.0304972 (46 \#4102)

[41] Robert S. Strichartz, Restrictions of Fourier transforms to quadratic surfaces and decay of solutions of wave equations, Duke Math. J. 44 (1977), no. 3, 705-714. MR.0512086 (58 \#23577)

[42] Gerald Teschl, Mathematical methods in quantum mechanics: With applications to Schrödinger operators, Graduate Studies in Mathematics, vol. 99, American Mathematical Society, Providence, RI, 2009. MR2499016(2010h:81002)

[43] Peter A. Tomas, A restriction theorem for the Fourier transform, Bull. Amer. Math. Soc. 81 (1975), 477-478. MR0358216 (50 \#10681)

[44] M. C. Vilela, Inhomogeneous Strichartz estimates for the Schrödinger equation, Trans. Amer. Math. Soc. 359 (2007), no. 5, 2123-2136 (electronic), DOI 10.1090/S0002-9947-06-04099-2. MR2276614 (2008a:35226)

[45] Claude Zuily, Uniqueness and nonuniqueness in the Cauchy problem, Progress in Mathematics, vol. 33, Birkhäuser Boston, Inc., Boston, MA, 1983. MR701544 (85d:35009)

Department of Mathematics, Sungkyunkwan University, Suwon 440-746, Republic of KOREA

E-mail address: ihseo@skku.edu 